\title{
High-resolution simulations and modeling of reshocked single-mode Richtmyer-Meshkov instability: Comparison to experimental data and to amplitude growth model predictions
}

\author{
Marco Latini ${ }^{\text {a) }}$ \\ Applied and Computational Mathematics, California Institute of Technology, Pasadena, California 91125 \\ Oleg Schilling ${ }^{\text {b) }}$ \\ University of California, Lawrence Livermore National Laboratory, Livermore, California 94551 \\ Wai Sun Don ${ }^{\mathrm{c})}$ \\ Division of Applied Mathematics, Brown University, Providence, Rhode Island 02912
}

(Received 30 November 2006; accepted 14 December 2006; published online 27 February 2007)

\begin{abstract}
The reshocked single-mode Richtmyer-Meshkov instability is simulated in two spatial dimensions using the fifth- and ninth-order weighted essentially nonoscillatory shock-capturing method with uniform spatial resolution of 256 points per initial perturbation wavelength. The initial conditions and computational domain are modeled after the single-mode, Mach 1.21 air(acetone)/SF 6 shock tube experiment of Collins and Jacobs [J. Fluid Mech. 464, 113 (2002)]. The simulation densities are shown to be in very good agreement with the corrected experimental planar laser-induced fluorescence images at selected times before reshock of the evolving interface. Analytical, semianalytical, and phenomenological linear and nonlinear, impulsive, perturbation, and potential flow models for single-mode Richtmyer-Meshkov unstable perturbation growth are summarized. The simulation amplitudes are shown to be in very good agreement with the experimental data and with the predictions of linear amplitude growth models for small times, and with those of nonlinear amplitude growth models at later times up to the time at which the driver-based expansion in the experiment (but not present in the simulations or models) expands the layer before reshock. The qualitative and quantitative differences between the fifth- and ninth-order simulation results are discussed. Using a local and global quantitative metric, the prediction of the Zhang and Sohn [Phys. Fluids 9, 1106 (1997)] nonlinear Padé model is shown to be in best overall agreement with the simulation amplitudes before reshock. The sensitivity of the amplitude growth model predictions to the initial growth rate from linear instability theory, the post-shock Atwood number and amplitude, and the velocity jump due to the passage of the shock through the interface is also investigated numerically. (C) 2007 American Institute of Physics. [DOI: 10.1063/1.2472508]
\end{abstract}

\section{INTRODUCTION}

The Richtmyer-Meshkov instability occurs when perturbations on the interface separating two different gases grow following the passage of a shock and eventually develop complex spatial structure. The instability derives its name from the linear instability analysis and numerical work of Richtmyer, ${ }^{1}$ who considered the instability generated by a shock impulsively accelerating a sinusoidally perturbed interface. The predictions of Richtmyer were subsequently confirmed in shock tube experiments performed by Meshkov. ${ }^{2}$ This instability is of great fundamental interest in fluid dynamics, ${ }^{3,4}$ as well as to inertial confinement fusion, ${ }^{5-8}$ supersonic combustion, ${ }^{9}$ and supernovae dynamics. ${ }^{10-14}$ One of the challenges in understanding the Richtmyer-Meshkov instability is the accurate modeling of the growth of the mixing layer in the nonlinear phase and following reshock, as

\footnotetext{
a)Electronic mail: mlatini@acm.caltech.edu

${ }^{\text {b) }}$ Author to whom correspondence should be addressed. Electronic mail: schilling1@1lnl.gov

${ }^{c)}$ Electronic mail: wsdon@cfm.brown.edu
}

well as predicting the statistical properties and dynamics of turbulent mixing induced by this instability.

In the present work, the evolution of the classical Richtmyer-Meshkov instability in a model of the air (seeded with acetone) and sulfur hexafluoride $\left(\mathrm{SF}_{6}\right)$ Mach 1.21 experiment of Collins and Jacobs ${ }^{15}$ is investigated in twodimensional planar geometry using the weighted essentially nonoscillatory (WENO) method. As the shock passes through the material interface separating the air and acetone mixture [referred to as air(acetone) in the sequel] and the denser sulfur hexafluoride gas, the misalignment of the pressure and density gradients causes a deposition of vorticity through the baroclinic production mechanism. The vorticity evolution equation (shown here for three dimensions and in the absence of molecular dissipation terms)

$$
\frac{\mathrm{d} \boldsymbol{\omega}}{\mathrm{d} t}=\frac{\boldsymbol{\nabla} \rho \times \nabla p}{\rho^{2}}+\boldsymbol{\omega} \cdot \nabla \mathbf{u}-\boldsymbol{\omega} \boldsymbol{\nabla} \cdot \mathbf{u}
$$

describes the dynamics of vorticity generation, where $\mathrm{d} / \mathrm{d} t$ $=\partial / \partial t+\mathbf{u} \cdot \boldsymbol{\nabla}$ is the convective derivative, $\boldsymbol{\omega}=\boldsymbol{\nabla} \times \mathbf{u}$ is the vorticity, $\mathbf{u}$ is the velocity, $\rho$ is the density, and $p$ is the 
pressure. The first term on the right side is the baroclinic production term, and constitutes the principal mechanism of vorticity generation by the Richtmyer-Meshkov instability. In particular, this term is large when a shock passes through the interface and when waves interact with the interface. The second term on the right side is the vortex-stretching term (which is zero in the present two-dimensional investigation, as the vorticity and velocity fields are orthogonal). This term enhances dissipation, resulting in more diffuse and smallerscale structures in three-dimensional turbulence. The third term on the right side is the compression term, and does not contribute significantly to the vorticity evolution. Following the passage of the shock, the perturbed interface is set in motion along the direction of shock propagation, a reflected shock returns back into the air(acetone) gas, and a transmitted shock enters the $\mathrm{SF}_{6}$ gas. The vorticity baroclinically deposited on the interface by the shock drives the evolution of the instability, with spikes of the heavier fluid $\left(\mathrm{SF}_{6}\right)$ penetrating the lighter fluid [air(acetone)] and bubbles of the lighter fluid "rising" in the heavier fluid. At late times, the vorticity coalesces into strong cores, causing the mushroom roll-ups characteristic of the Richtmyer-Meshkov instability. When the fluids are miscible, molecular mixing occurs between these interpenetrating fluids, generating a mixing layer and a topologically complex interface. In the present investigation, explicit molecular mixing is not modeled; instead, numerical diffusion across the interface models the "mixing" process.

Additional vorticity deposition and enhanced mixing occur in the configuration considered here, in which the transmitted shock from the initial shock-interface interaction reflects elastically from the end wall of the shock tube test section and interacts with the evolving layer. This second interaction (referred to as reshock) deposits vorticity of opposite sign so that bubbles transform into spikes and vice versa in a process called inversion. The inversion induces the formation of additional complex structure, with more disorganized small-scale flow features observed at late times. Following reshock, the interface changes its direction of motion and moves away from the end wall of the test section: a transmitted shock enters the air(acetone) and a reflected rarefaction returns into the $\mathrm{SF}_{6}$. The rarefaction wave is reflected from the end wall of the test section and interacts with the evolving interface, again resulting in the formation of additional complex, small-scale structures.

Throughout the instability evolution, both prior to and following reshock, additional vortex-accelerated secondary baroclinic vorticity is deposited on the interface, ${ }^{3,16}$ where the vorticity is generated by the misalignment between the density gradient and the centripetal acceleration of the cores $\nabla p \times \nabla \rho \sim-\rho \mathrm{d} \mathbf{u} / \mathrm{d} t \times \nabla \rho$. This secondary instability further enhances the development of a complex interface topology (with increasing length in two dimensions and surface area in three dimensions), eventually resulting in a mixing layer that becomes turbulent at sufficiently large Reynolds numbers. ${ }^{17}$ The single-mode Richtmyer-Meshkov instability is considered in the present work to investigate the linear and nonlinear phases prior to reshock.

To our knowledge, the present work is the first compre- hensive application of the WENO method to the study of the classical reshocked Richtmyer-Meshkov instability. The vast majority of numerical simulations and experiments on single- and multimode Richtmyer-Meshkov instabilities to date have considered the flow evolution initiated by only a single shock-interface interaction. Mikaelian ${ }^{18}$ performed arbitrary Lagrangian-Eulerian simulations of gas configurations consisting of three layers, $1 / 2 / 1$, with fluid 1 representing semi-infinite air layers and fluid 2 representing a finite-thickness freon, $\mathrm{SF}_{6}$, or helium layer, having perturbations either on the upstream or downstream side. The shock Mach number was 1.5. These simulations investigated freeze-out, interface coupling, and feedthrough. Sadot et al. ${ }^{19}$ found very good agreement between the amplitude growth from their single-mode reshocked Richtmyer-Meshkov instability experiment and numerical simulation data prior to the arrival of the rarefaction wave from the end wall. As in the case of experiments, the quantitative data obtained from these simulations were mainly limited to the consideration of perturbation amplitude growth. Numerical studies of the reshocked single-mode impulsive Richtmyer-Meshkov instability experiment of Jacobs, Jones, and Niederhaus ${ }^{20,21}$ were performed by Kotelnikov and Zabusky ${ }^{22}$ and Kotelnikov, Ray, and Zabusky ${ }^{23}$ using the vortex-in-cell method and the contour advection semi-Lagrangian method (n.b., the Jacobs et l. $^{24}$ and Rightley et $a .^{25}$ Mach 1.2 experiment with reshock was also simulated using a Godunov method ${ }^{23}$ ). Kremeyer $e t a l .^{26}$ used a fifth-order WENO method to simulate the Richtmyer-Meshkov instability in a shock tube containing gases with different initial transverse density profiles to investigate shock splitting and, in particular, the role of shock bowing and vorticity dynamics. Top-hat shaped perturbations, including those shaped as a notch, were considered instead of a sinusoidal perturbation considered in classical investigations of this instability. Zhang et al. ${ }^{27}$ simulated the interactions between planar Mach 1.095 and 1.2 shocks and an $\mathrm{SF}_{6}$ gas cylinder using the fifth- and seventh-order WENO method, and qualitatively and quantitatively studied the mechanisms of baroclinic vorticity and circulation generation. All of the above simulations were two-dimensional.

The key new contributions to better understanding the two-dimensional reshocked Richtmyer-Meshkov instability presented here are (i) the first simulations of the reshocked Richtmyer-Meshkov instability using high-order WENO methods; (ii) a comprehensive survey of a wide range of amplitude growth models and a summary of their derivations and phenomenology to investigate the nonlinear phase of this instability; (iii) direct qualitative and quantitative comparisons of simulation data to experimental data (thus providing an element of validation of the numerical methods as used here) and discussion of the differences between the fifth- and ninth-order WENO results; (iv) a comprehensive application of the amplitude growth models to one experimental and simulation dataset (also with the bubble and spike amplitudes obtained separately by performing an unperturbed simulation); and (v) a sensitivity study to emphasize that small differences in the amplitude model parameters can affect the model predictions.

This paper is organized as follows. The equations solved 
and a summary of the WENO method are presented in Sec. II A. The initial conditions and computational domain adapted from the Mach 1.21 experiment of Collins and Jacobs are specified in Sec. II B. Simulations of the reshocked single-mode Richtmyer-Meshkov instability using the fifthand ninth-order WENO method are presented and discussed in Sec. II C. The density evolution is compared to the corrected experimental PLIF images of Collins and Jacobs before and after reshock in Sec. II D. A review of the principal linear and nonlinear models for single-mode perturbation amplitude growth is presented in Sec. III, including the most widely used impulsive, perturbation, and potential flow models. The simulation amplitudes before reshock are compared to the experimental data and to the model predictions in Sec. IV. Finally, the sensitivity of the amplitude growth model predictions to the parameters entering into the models (the initial linear growth rate, the post-shock Atwood number, the post-shock amplitude, and the velocity jump due to the passage of the shock through the interface) is explored by selfconsistently comparing the predictions using numerically and experimentally determined values of these parameters. A summary of the results and conclusions are given in Sec. V.

\section{HIGH-RESOLUTION SIMULATIONS OF THE TWO-DIMENSIONAL RESHOCKED SINGLE-MODE RICHTMYER-MESHKOV INSTABILITY}

In this section, weighted essentially nonoscillatory (WENO) shock-capturing simulations of two-dimensional reshocked single-mode Richtmyer-Meshkov instability are described. The WENO method is a modern high-resolution reconstruction-evolution shock-capturing method ${ }^{28,29}$ used for the simulation of compressible turbulent flows. As the numerical algorithm is based on the discretization of the Euler equations, the truncation errors can be regarded as an implicit nonlinear high-order numerical dissipation, i.e., no explicit artificial viscosity or filtering to suppress Gibbs oscillations is required. As the nondissipative compressible fluid dynamics equations are formally ill-posed, ${ }^{30}$ this numerical dissipation regularizes the scheme and renders the method stable. Formally higher-order numerical flux reconstructions are less dissipative than lower-order reconstructions. It is important to appreciate that flows with shocks cannot be modeled using direct numerical simulation (DNS), and are therefore presently modeled using numerically dissipative shock-capturing schemes, monotone-integrated largeeddy simulations $^{31}$ (MILES), or implicit large-eddy simulations $^{32}$ (ILES). It is also important to appreciate that two-dimensional simulations can achieve much higher resolutions than can be obtained in three dimensions using the same computational resources. In a separate detailed study ${ }^{33}$ of the two-dimensional Mach 1.21 Collins and Jacobs experiment investigating the effects of order of WENO flux reconstruction and grid resolution, it was shown that higherorder WENO schemes on coarser grids are more computationally efficient in obtaining the desired resolution of relevant physical quantities than lower-order WENO schemes on finer grids.

\section{A. Equations solved and the WENO method}

The characteristics-based weighted essentially nonoscillatory (WENO) method in the conservative finite-difference formulation $^{34,35}$ is used in the present investigation. The Euler equations are augmented by the equation for mass fraction conservation of the second gas $\left(\mathrm{SF}_{6}\right)$ in order to track the interface and mixing: in two dimensions

$$
\frac{\partial}{\partial t}\left[\begin{array}{c}
\rho \\
\rho u \\
\rho v \\
\rho e \\
\rho m
\end{array}\right]+\frac{\partial}{\partial x}\left[\begin{array}{c}
\rho u \\
\rho u^{2}+p \\
\rho u v \\
(\rho e+p) u \\
\rho m u
\end{array}\right]+\frac{\partial}{\partial y}\left[\begin{array}{c}
\rho v \\
\rho u v \\
\rho v^{2}+p \\
(\rho e+p) v \\
\rho m v
\end{array}\right]=0,
$$

where $\rho$ is the density, $\mathbf{u}=(u, v)$ is the velocity, $p$ is the pressure, $e=\left(u^{2}+v^{2}\right) / 2+U$ is the total (kinetic plus internal) energy per unit mass, $U=p /[\rho(\gamma-1)]$ is the internal energy, $p=\rho R T$ is the ideal gamma law gas pressure ( $R$ is the universal gas constant), and $m$ is the mass fraction (here of $\mathrm{SF}_{6}$ ). In the present simulations, a single gamma is used as multiple gammas create nonphysical pressure oscillations near the material interfaces (which are nontrivial to mitigate) in conservative shock-capturing schemes for the multicomponent fluid equations. ${ }^{36-39}$ The fifth- and ninth-order simulations were identical in every respect except for the reconstruction order.

A conservative finite-difference formulation for hyperbolic conservation laws requires consistent high-order numerical fluxes at the cell boundaries in order to construct the flux difference across the uniformly spaced cells. For the Euler equations, the eigensystem of fluxes is obtained from the Jacobian of the Roe-averaged fluxes in each spatial dimension. Lax-Friedrichs flux-splitting is used to split the original fluxes into their positive and negative components (with an introduction of additional artificial dissipation in order to obtain smoother fluxes ${ }^{34}$ ). The resulting positive and negative flux components are then projected in the characteristic fields using the left eigenvectors to form the positive and negative characteristic variables at each cell center. Then, WENO polynomial reconstruction is used to reconstruct the fluxes at the cell boundaries with a high order of accuracy: a weighted convex combination of all possible $r$ th degree piecewise-polynomial approximations (e.g., $r=3$ for fifth-order and $r=5$ for ninth-order) of the characteristic variables using the neighboring cell-centered values is constructed and evaluated at the boundaries of a given cell. For a given order of reconstruction, there are $r$ possible $r$ th-degree piecewise polynomials available, with smoothness properties depending on the smoothness of the underlying solution. The essential element of WENO reconstruction is an adaptive nonlinear assignment of the weights given to those $r$ polynomials: the weight is designed such that a polynomial of degree $2 r-1$ is formed for the locally smooth regions of the solution, and an essentially nonoscillatory $r$ th-degree polynomial (i.e., upwinding) is used otherwise to avoid Gibbs oscillations when approximating solution discontinuities. The weights of the $r$ choices of possible stencils around a given cell center are computed utilizing the prop- 
erly scaled local gradient and curvature of the data via a divided difference. Hence, relatively smaller (or close to zero) weights are assigned to polynomials approximating the discontinuous data and nearly equal weights are assigned to polynomials approximating the smooth data. The high-order reconstructed positive and negative characteristic variables are summed and then projected back to physical space via the right eigenvectors, forming a high-order numerical flux at the cell boundaries. The formal spatial order of accuracy of the scheme is $2 r-1$ in smooth flow regions. As for all other shock-capturing methods, the actual computational accuracy diminishes to at best first-order near the shock. Although the global order of the solution is reduced to first near a shock, the high-order reconstruction yields an efficient highresolution algorithm, which is essential to long-time, multiscale simulations of complex shock-induced flows. In the present study, the semidiscrete equations are evolved in time using the third-order total variation diminishing (TVD) Runge-Kutta scheme. ${ }^{40}$

The WENO code used here provides a framework for the multidimensional numerical simulation of the fully nonlinear evolution of hydrodynamic instabilities and mixing generated by single- and multimode Richtmyer-Meshkov, Rayleigh-Taylor, and Kelvin-Helmholtz instabilities. The nonlinear system of hyperbolic partial differential equations can be solved in one, two, or three spatial dimensions. Although fifth- and ninth-order flux reconstructions are used presently, other odd orders of WENO polynomial reconstruction can be specified, including third-, seventh-, and eleventh-order.

\section{B. Description of the Mach 1.21 experiment of Collins and Jacobs}

The initial conditions for the present simulations were adapted from the Mach 1.21 shock tube experiment of Collins and Jacobs. ${ }^{15}$ This experiment was selected for comparison of the numerical results to the high-resolution corrected density planar laser-induced fluorescence (PLIF) images showing the evolution of the instability and to the measured perturbation amplitude prior to reshock. The experiment was performed in a vertical shock tube using a novel technique to generate a membraneless perturbed interface. The shock tube had a square test section with cross section $8.9 \mathrm{~cm}$ $\times 8.9 \mathrm{~cm}$ and length $75 \mathrm{~cm}$. The driver section had a diameter of $10.2 \mathrm{~cm}$ and length $100 \mathrm{~cm}$ (with a mixture of 50\% air and $50 \% \mathrm{~N}_{2}$ ), and the driven section has a cross section of $8.9 \mathrm{~cm} \times 8.9 \mathrm{~cm}$ and a length of $261 \mathrm{~cm}$ (n.b., these dimensions are specified more accurately than originally reported by Collins and Jacobs ${ }^{15}$ ). The shock was generated through the rupture of a diaphragm and propagated into a mixture of $75 \%$ air and $25 \%$ acetone by volume at standard room temperature and pressure. The shock then refracted at the perturbed interface separating the air(acetone) mixture and the denser sulfur hexafluoride $\left(\mathrm{SF}_{6}\right)$ gas, giving rise to the growth and evolution of the Richtmyer-Meshkov instability.

The membraneless interface was generated as follows. ${ }^{41}$ The shock tube contained horizontal slots on the two opposite walls. The gases entered the shock tube from the oppo- site ends, flowed toward each other, and exited through the two slots, resulting in a fine, initially diffuse interface separating the air(acetone) and $\mathrm{SF}_{6}$. A sinusoidal perturbation was then generated by gently oscillating the vertical shock tube horizontally using an eccentric stepper motor at a prescribed frequency to establish a standing wave. The initial diffuse interface was estimated to be $\approx 0.5 \mathrm{~cm}$ wide using the maximum slope determined by the measurement of the acetone concentration. PLIF was used to visualize the instability evolution using a mixture of the fluorescent acetone with air, and the images were corrected for nonuniform laser illumination and Beer's law attenuation. A set of experiments was conducted for shocks with Mach numbers 1.21 \pm 0.02 , and images were captured up to $11 \mathrm{~ms}$ following the initial shock-interface interaction. The evolution of the instability, with spikes of heavier fluid penetrating the lighter fluid and bubbles of lighter fluid "rising" in the heavier fluid, was investigated. The reshock phase, when the transmitted shock reflected from the end wall of the test section and interacted with the evolving interface, was also described. Prior to the arrival of the reflected shock, a reflected rarefaction wave also interacted with the interface. This initial rarefaction wave (referred to as the driver-based expansion wave in the sequel) was created by the rupture of the membrane generating the initial shock; this wave was subsequently reflected from the end (top) wall of the shock tube, and then interacted with the interface. This interaction with the reflected rarefaction formed additional complex structure on the evolving interface.

\section{Description of the simulations}

In the present simulations, only the shock tube test section is considered, so that the driver-based expansion wave is not captured numerically. Furthermore, the effects of gravity and the horizontal motion of the shock tube are neglected. The upstream conditions were matched in order to specify a single value of the adiabatic exponent, $\gamma=1.276$, corresponding to the air(acetone) mixture. The thermodynamic properties of air(acetone) vapor and $\mathrm{SF}_{6}$, including differences between the single-gamma simulations and the conditions that would be obtained if two gammas were used, are given in Table I (where some quantities were obtained using onedimensional shock refraction theory). Reshock occurs $\approx 0.25 \mathrm{~ms}$ earlier in the present simulations than would occur in two-gamma simulations.

To match the shock tube test section dimensions, the computational domain had $(x, y)$ dimension $\left[0, L_{x}\right] \times\left[0, L_{y}\right]$ with $L_{x}=78 \mathrm{~cm}, L_{y}=8.9 \mathrm{~cm}$, and centerline of the perturbed initial interface located at $x=3 \mathrm{~cm}$. The shock is initiated at $x=1 \mathrm{~cm}$ using the Rankine-Hugoniot conditions corresponding to a $\mathrm{Ma}=1.21$ shock in the air(acetone). The simulations had a grid resolution $\Delta x=\Delta y=0.0232 \mathrm{~cm}$ corresponding to 256 points per initial perturbation wavelength. A CFL number of 0.45 was used.

As in the experiment, the sinusoidal interfacial perturbation in the simulations $\eta(y)=a_{0} \sin (k y)$ had amplitude $a_{0}$ $=0.2 \mathrm{~cm}$ (slightly larger than the mean value $0.183 \mathrm{~cm}$ reported in the experiment) to better match the post-shock am- 
TABLE I. The pre- and post-shock state [adiabatic exponents $\gamma_{r}$, molecular weights $M_{r}$, incident, reflected, and transmitted shock speeds $u_{i, \text { shock }}, u_{r, \text { shock }}$, and $u_{t, \text { shock }}$, densities $\rho_{r}$, Atwood number $A=\left(\rho_{\mathrm{SF}_{6}}-\rho_{a a}\right)\left(\rho_{\mathrm{SF}_{6}}+\rho_{a a}\right)$, pressure $p$, and temperatures $\left.T_{r}(r=1,2)\right]$ of the air(acetone) and $\mathrm{SF}_{6}$ gases from one-dimensional shock refraction theory. The simulation (single-gamma) values were obtained assuming $\gamma=1.276$ and the two-gamma values were obtained using $\gamma_{1}=1.276$ and $\gamma_{2}=1.093$, corresponding to the air(acetone) mixture and the $\mathrm{SF}_{6}$, respectively. The time of reshock, $t_{\text {reshock }}$, is also indicated.

\begin{tabular}{|c|c|c|c|c|}
\hline & \multicolumn{2}{|c|}{ Single-gamma (simulation) value } & \multicolumn{2}{|c|}{ Two-gamma value } \\
\hline$\gamma_{\text {aа }}$ & \multicolumn{2}{|c|}{1.276} & \multicolumn{2}{|c|}{1.276} \\
\hline$\gamma_{\mathrm{SF}_{6}}$ & \multicolumn{2}{|c|}{1.276} & \multicolumn{2}{|c|}{1.093} \\
\hline$M_{\text {aа }}(\mathrm{g} / \mathrm{mol})$ & \multicolumn{2}{|c|}{34.76} & \multicolumn{2}{|c|}{34.76} \\
\hline$M_{\mathrm{SF}_{6}}(\mathrm{~g} / \mathrm{mol})$ & \multicolumn{2}{|c|}{146.05} & \multicolumn{2}{|c|}{146.05} \\
\hline$u_{i, \text { shock }}(\mathrm{cm} / \mathrm{s})$ & \multicolumn{2}{|c|}{36369} & \multicolumn{2}{|c|}{36369} \\
\hline$u_{r, \text { shock }}(\mathrm{cm} / \mathrm{s})$ & \multicolumn{2}{|c|}{33473} & \multicolumn{2}{|c|}{33301} \\
\hline$u_{t, \text { shock }}(\mathrm{cm} / \mathrm{s})$ & \multicolumn{2}{|c|}{19218} & \multicolumn{2}{|c|}{17945} \\
\hline \multirow[t]{2}{*}{$t_{\text {reshock }}(\mathrm{ms})$} & \multicolumn{2}{|c|}{6.36} & \multicolumn{2}{|c|}{6.61} \\
\hline & Pre-shock & Post-shock & Pre-shock & Post-shock \\
\hline$\rho_{\text {aa }}\left(\mathrm{g} / \mathrm{cm}^{3}\right)$ & $1.351 \times 10^{-3}$ & $2.084 \times 10^{-3}$ & $1.351 \times 10^{-3}$ & $2.065 \times 10^{-3}$ \\
\hline$\rho_{\mathrm{SF}_{6}}\left(\mathrm{~g} / \mathrm{cm}^{3}\right)$ & $5.494 \times 10^{-3}$ & $8.456 \times 10^{-3}$ & $5.494 \times 10^{-3}$ & $9.021 \times 10^{-3}$ \\
\hline$A$ & 0.6053 & 0.6045 & 0.6053 & 0.6274 \\
\hline$p$ (bar) & 0.956 & 1.667 & 0.956 & 1.648 \\
\hline$T_{\text {aа }}(\mathrm{K})$ & 296 & 334.4 & 296 & 333.6 \\
\hline$T_{\mathrm{SF}_{6}}(\mathrm{~K})$ & 305 & 346 & 305 & 321 \\
\hline
\end{tabular}

plitude, perturbation wavelength $\lambda=5.93333 \mathrm{~cm}$, and diffuse interface width $\delta=0.5 \mathrm{~cm}$. Thus, $k a_{0}=0.21 \ll 1$, where $k$ $=2 \pi / \lambda$ is the perturbation wave number, so that the initial growth is linear.

The boundary conditions were (i) inflow at the entrance of the test section in the streamwise $(x)$ direction; (ii) reflecting at the end wall of the test section in the streamwise direction, where $u(x, y, t)=-u(x, y, t)$, and; (iii) symmetry in the spanwise ( $y$ ) direction corresponding to the cross section of the test section, where $\phi(-x,-y, t)=\phi(x, y, t)$ and $\phi$ $=(\rho, u, v, p, m)^{T}$. Any turbulent boundary layers on the side walls or other molecular viscous/diffusive effects present in the experiment are not captured in these inviscid simulations.

In the current investigation, where the computed fluid dynamic fields remain unchanged over large regions of the domain until late times, a novel adaptive domain algorithm automatically enlarged the domain size along the shock propagation direction, when changes from the constant state were detected. This allowed the simulation to begin with a smaller domain of length $L_{x}=9.3472 \mathrm{~cm}$, corresponding to a streamwise resolution of $N_{x}=407$. When the algorithm detected changes in a designated quantity (such as density in the present case), as the shock or disturbances arrived near the downstream shortened domain boundary, the domain size was enlarged by $3 \mathrm{~cm}$ until the end of the domain was reached at $L_{x}=78 \mathrm{~cm}$, corresponding to a final resolution of $N_{x}=3372$.

The simulations were performed using a parallel Fortran 90 code on the Blue-Pacific computer at the Lawrence Livermore National Laboratory; each processor is a PowerPC $604 \mathrm{e}$ with $1.5 \mathrm{~GB}$ per 4-CPU node and clock speed of $332 \mathrm{MHz}$. For each simulation, 32 nodes were used with a total of 128 processors.

\section{Qualitative comparison of density evolution to experimental PLIF images}

Corrected PLIF density images from the Collins and Jacobs Mach 1.21 shock tube experiment are compared to the simulation density fields in Fig. 1 at selected times (2.5, 3, 4,

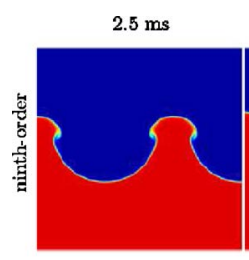

$3 \mathrm{~ms}$

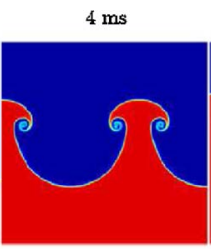

$5 \mathrm{~ms}$
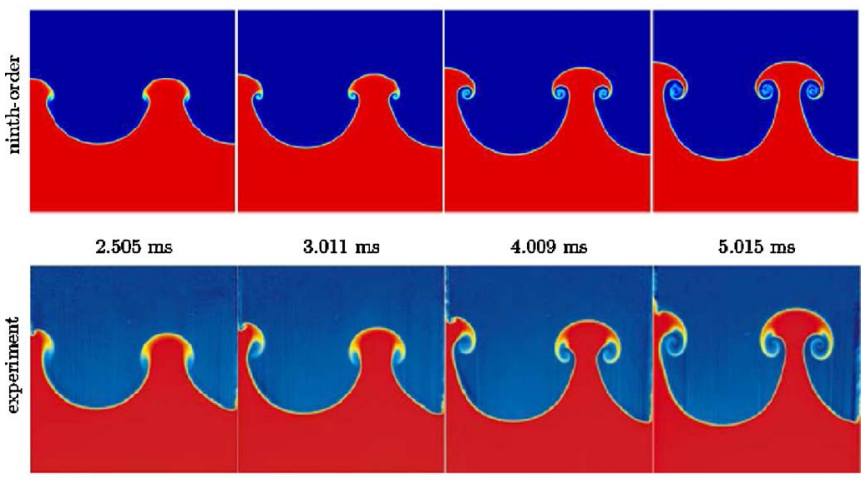

$2.5 \mathrm{~ms}$

$3 \mathrm{~ms}$

$4 \mathrm{~ms}$

$5 \mathrm{~ms}$

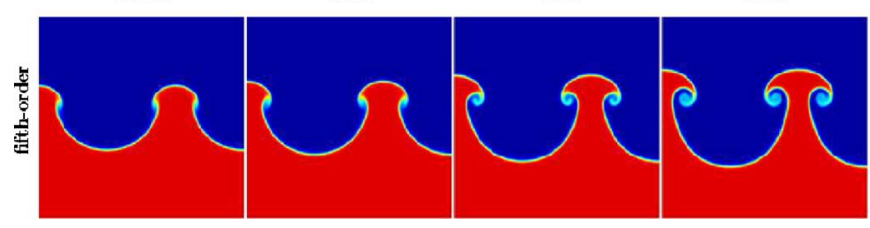

FIG. 1. (Color online) Comparison of corrected PLIF images from the Collins and Jacobs (Ref. 15) experiment (middle row) with the density from the ninth-order WENO simulation (top row) and from the fifth-order WENO simulation (bottom row) at selected times before reshock. The gases are air(acetone) (top) and $\mathrm{SF}_{6}$ (bottom). The simulation times differ slightly from the experimental times by $0.005-0.015 \mathrm{~ms}$. The experimental images are taken from Fig. 6 of Collins and Jacobs (reprinted with the permission of Cambridge University Press). 

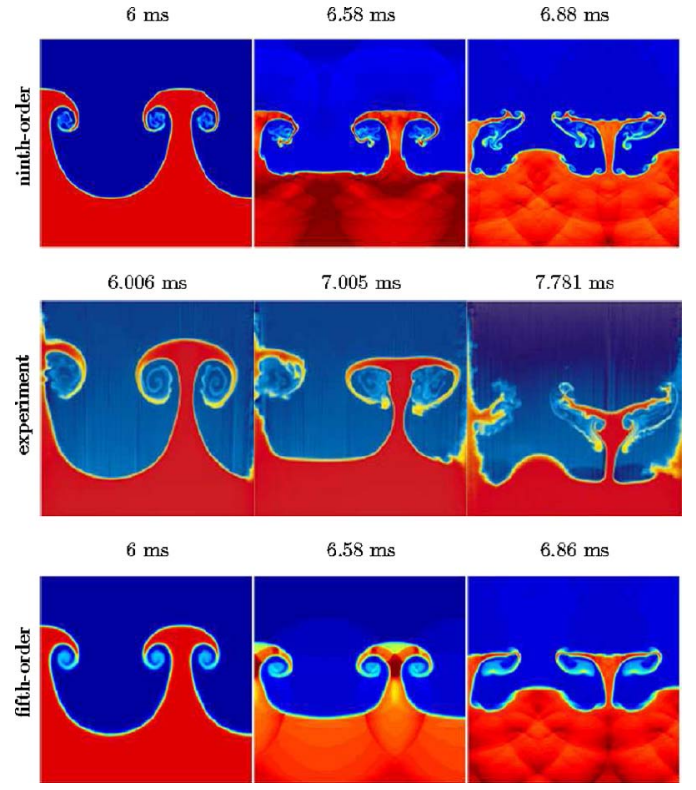

FIG. 2. (Color online) Further comparison of corrected PLIF images from the Collins and Jacobs (Ref. 15) experiment and the density from the fifthand ninth-order WENO simulations at selected times just before and after reshock of the evolving interface. The experimental images are taken from Fig. 6 of Collins and Jacobs (reprinted with the permission of Cambridge University Press).

and $5 \mathrm{~ms}$ ) before reshock: the experimental images are shown in the middle row and the fields from the ninth- and fifth-order simulation are shown in the top and bottom row, respectively (n.b., the simulation fields are rotated $90^{\circ}$ counterclockwise in Figs. 1-3 to facilitate comparison with the experimental images). The PLIF images are shown in false color, with red and blue corresponding to the lowest and highest fluorescent intensity, respectively. The temporal sequence of images was obtained from an ensemble of highly reproducible shock tube experiments. In general, the numerical and experimental images are in very good qualitative agreement. By $5 \mathrm{~ms}$, a well-formed mushroom roll-up forms with entrainment of the air(acetone) into the vortex core. Small differences in the width of the mushroom "stem" and the width of the "cap" can be observed between the experiment and the simulations. The experimental images show more pronounced structure inside the vortex roll-ups, and the

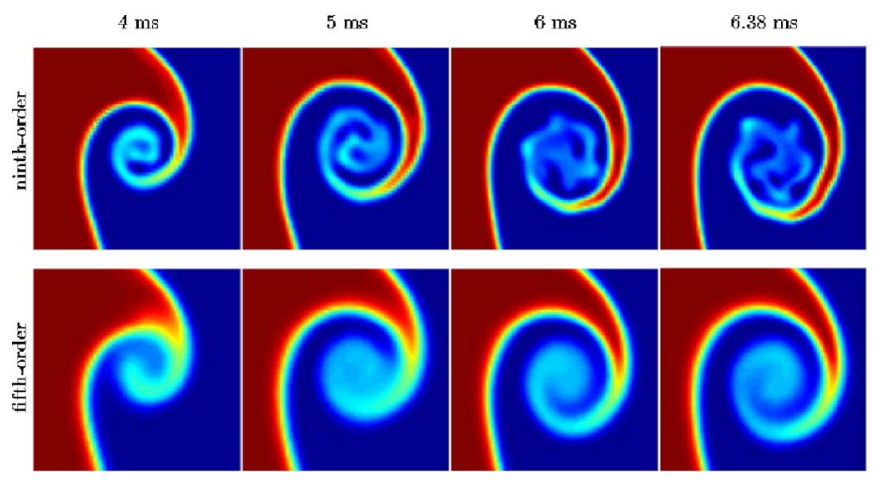

FIG. 3. (Color online) Details of the roll-up in the density field, $\rho$, from the ninth-order (top row) and fifth-order (bottom row) WENO simulations at 4 , 5,6 , and $6.38 \mathrm{~ms}$. roll-ups in the simulations are more spatially compact than those in the experiment. The ninth-order simulation captures more of the roll-up structure observed in the experiment at late times than does the fifth-order simulation.

The comparison of the experimental PLIF images to the simulation densities is continued in Fig. 2 at times just before and following reshock (6, 6.58, and $6.88 \mathrm{~ms})$. The ninthorder simulation captures the secondary instability within the roll-ups at $6 \mathrm{~ms}$. The simulation densities at 6.58 and $6.88 \mathrm{~ms}$ exhibit the wave structure developing at the interface as the transmitted shock enters the air(acetone) and the reflected rarefaction returns into the $\mathrm{SF}_{6}$. A progressively larger time delay reaching $\approx 1 \mathrm{~ms}$ develops by $6.88 \mathrm{~ms}$ as the simulations lag in time behind the PLIF density images. This time delay can be explained by the arrival of the driverbased expansion wave in the experiment (which decelerates the evolving interface), as well as by the difference in the interface velocity between the simulations and experiment. The rarefaction also causes the formation of small-scale structures on the interface that are amplified during reshock. The experimental image shows increased mixing and fragmentation of large structures at $7.781 \mathrm{~ms}$ : this results from the excitation of fluctuations in the third spatial dimension caused by the driver-based expansion wave in the experiment and subsequently amplified by reshock (which is not captured in the simulations), as well as by other possible excitation mechanisms. The simulations show that more complex, finer, and disordered structures form following reshock. In addition, the ninth-order simulation shows the appearance of small-scale roll-ups on the fluid interface that are not present in the experimental and fifth-order simulation images. This may be due to molecular dissipation effects in the experiment, and to the numerical dissipation in the fifthorder simulation that is significantly larger than that in the ninth-order simulation. Note that the PLIF images in Figs. 1 and 2 exhibit boundary layer effects on the left side wall that are not present in the simulations.

A close-up of the roll-ups, including a comparison of the internal structure from the fifth- and ninth-order simulations, is shown in Fig. 3 at 4, 5, 6, and $6.38 \mathrm{~ms}$. The large-scale features are similar between the simulations. The ninth-order simulation densities exhibit sharper roll-ups and include more complex internal corrugated structure than the fifthorder densities. The additional structure within the roll-up has been attributed to the vortex-accelerated secondary baroclinic circulation, ${ }^{16}$ which causes vorticity of opposite sign to appear inside the core, contributing to additional mixing and to fragmentation. Such structures have also been observed in the late-time, single-mode Richtmyer-Meshkov instability experiments of Jacobs and Krivets. ${ }^{42}$

The qualitative comparison above shows that it is possible to achieve very good agreement between a twodimensional, high-resolution shock-capturing simulation with high-order WENO flux reconstruction and experimental density PLIF images before reshock when three-dimensional effects are not significant. In addition, the comparison of the densities from the fifth- and ninth-order simulations at 5 and $6 \mathrm{~ms}$ demonstrates that higher-order reconstruction better captures secondary instabilities on the interface and within 
the vortex cores. The roll-ups in the ninth-order simulation are tighter and sharper, and have more fine-scale structure. The simulation fields also display the shock focusing during the reshock process.

Following reshock, the experiment and simulations exhibit distinctively different flow structures due to the absence of the driver-based expansion wave and the increased importance of three-dimensional effects (including vortex stretching and associated mechanisms) that are not accounted for in the present simulations. This results in the formation and persistence of large-scale structures in the simulations, consistent with the inverse cascade of kinetic energy from small scales to larger scales in two-dimensional turbulence experiments and simulations, and explains the much larger simulation amplitudes following reshock compared to the experimental data points. The experiments are affected by molecular diffusion and dissipation mechanisms that are not explicitly modeled by the equations solved numerically, i.e., the simulations contain implicit numerical diffusion and dissipation that may not be comparable to the molecular values in the physical experiment. The comparison of the fifth- and ninth-order simulations shows that as the order is increased, finer and more complex asymmetric structures appear, consistent with a reduced level of numerical dissipation. The sensitivity of the results to third-, fifth-, and ninth-order spatial flux reconstruction, to grid resolution corresponding to 128,256 , and 512 points per initial perturbation wavelength, and to numerical dissipation are systematically and selfconsistently examined elsewhere. ${ }^{33}$

\section{LINEAR AND NONLINEAR PERTURBATION AMPLITUDE GROWTH MODELS}

The prediction of Richtmyer-Meshkov instability growth in the weakly nonlinear, nonlinear, and turbulent regimes is of contemporary interest. ${ }^{3,4}$ An overview of the principal perturbation amplitude growth models categorized according to their underlying physical assumptions on the flow is presented in this section. It is implicitly assumed in these models that molecular dissipation and diffusion effects, surface tension, and other effects are negligible (extensions of some of the models to include such effects have been developed, but are not discussed here). While each of these models has limitations and a restricted domain of applicability, they represent an effort to better understand fundamental aspects of Richtmyer-Meshkov instability growth into the nonlinear regime.

The definitions of the bubble and spike amplitudes, $a_{b}(t)$ and $a_{s}(t)$, and the mixing layer width $h(t)$ are illustrated in Fig. 4. The contour shows the canonical early-time evolution of the interface induced by the Richtmyer-Meshkov instability from a sinusoidal initial perturbation. The spikes penetrate into the lighter fluid and roll up, while bubbles "rise" into the heavier fluid. The solid horizontal line shows the location of the shocked, unperturbed interface used as a reference for the determination of the bubble and spike amplitudes from the simulation data. The distance from the unperturbed interface to the bubble tip is the bubble amplitude $a_{b}$, while the distance from the unperturbed interface to the spike

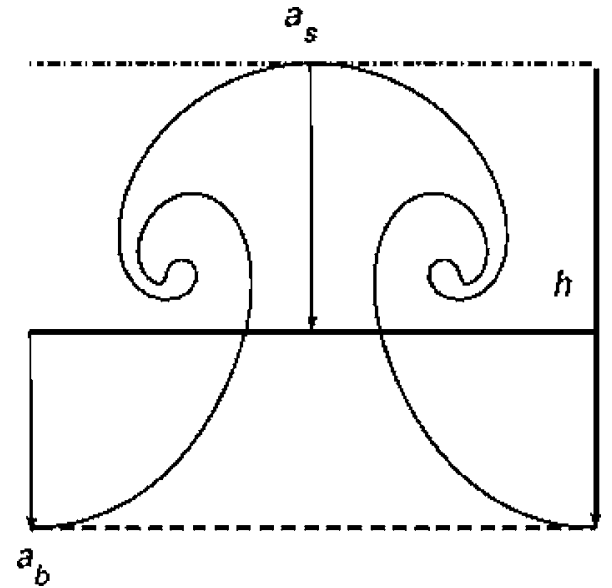

FIG. 4. A typical interface evolving according to the single-mode Richtmyer-Meshkov instability with bubble and spike amplitudes $a_{b}$ and $a_{s}$, and mixing layer width $h$ (the image is from a point vortex simulation). The solid line in the center is the location of the shocked unperturbed interface, and is used as the reference to measure the bubble and spike amplitudes.

tip is the spike amplitude $a_{s}$. The numerical determination of these amplitudes is discussed in Sec. IV A. The mixing layer width is the sum of the bubble and spike amplitudes

$$
h(t)=a_{b}(t)+a_{s}(t) .
$$

The mixing layer amplitude predicted by the models in this section is the average of the bubble and spike amplitudes,

$$
a(t)=\frac{a_{b}(t)+a_{s}(t)}{2},
$$

i.e., one-half the mixing layer width. The corresponding mixing layer width and amplitude growth rates are $\mathrm{d} h / \mathrm{d} t$ $=\mathrm{d} a_{b} / \mathrm{d} t+\mathrm{d} a_{s} / \mathrm{d} t$ and $\mathrm{d} a / \mathrm{d} t=\left(\mathrm{d} a_{b} / \mathrm{d} t+\mathrm{d} a_{s} / \mathrm{d} t\right) / 2$, respectively. Each of the models for $\mathrm{d} a / \mathrm{d} t$ considered below can be integrated analytically to obtain $a(t)$. The corresponding bubble and spike (tip) velocities used in Sec. IV C are $v_{b}(t)=\mathrm{d} a_{b} / \mathrm{d} t$ and $v_{s}(t)=\mathrm{d} a_{s} / \mathrm{d} t$, respectively.

For the model summary given below, it is useful to define the key quantities appearing in the models: $k=2 \pi / \lambda$ is the wave number of the initial perturbation (where $\lambda$ is the initial perturbation wavelength), $a_{0}^{-}$is the pre-shock initial perturbation amplitude, $a_{0}^{+}$is the post-shock initial perturbation amplitude, $A^{-}=\left(\rho_{1}^{-}-\rho_{2}^{-}\right) /\left(\rho_{1}^{-}+\rho_{2}^{-}\right)$is the pre-shock Atwood number, $A^{+}=\left(\rho_{1}^{+}-\rho_{2}^{+}\right) /\left(\rho_{1}^{+}+\rho_{2}^{+}\right)$is the post-shock Atwood number, and $\Delta u$ is the velocity jump at the interface following shock refraction. In some of the definitions of the models, the scalings

$$
\tau=k v_{0} t, \quad v_{0}=k \Delta u A^{+} a_{0}^{+}
$$

are used, where $v_{0}$ is the Richtmyer velocity.

\section{A. Impulsive models}

Impulsive models based on representing the shock as an instantaneous $\delta$-function acceleration are briefly reviewed and summarized here. Impulsive models for the RichtmyerMeshkov instability were developed by adapting existing models for the Rayleigh-Taylor instability (subject to a con- 
stant acceleration) to the case of an impulsive acceleration. These models predict a linear growth in time of the perturbation amplitude that captures the early stages of the instability evolution before nonlinear saturation effects become important.

\section{The Richtmyer model}

The first impulsive model proposed for the growth of a single-mode perturbation is due to Richtmyer. ${ }^{1}$ Richtmyer modified earlier work ${ }^{43}$ on the growth of a small single-mode perturbation with amplitude $a(t)$ and wave number $k$ when a dense fluid is accelerated continuously into a lighter fluid [the Rayleigh-Taylor instability described by $\mathrm{d}^{2} a / \mathrm{d} t^{2}=g A k a$ with $A=\left(\rho_{1}-\rho_{2}\right) /\left(\rho_{1}+\rho_{2}\right)$ the Atwood number], by replacing the constant gravitational acceleration $g$ with an impulsive acceleration $\Delta u \delta(t)$,

$$
\frac{\mathrm{d} a}{\mathrm{~d} t}=k \Delta u A^{+} a_{0}^{+} \equiv v_{0} .
$$

Integrating gives

$$
a(t)=\left(1+k \Delta u A^{+} t\right) a_{0}^{+} .
$$

\section{The Meyer-Blewett model}

Meyer and Blewett ${ }^{44}$ performed two-dimensional Lagrangian simulations of the single-mode RichtmyerMeshkov instability and computed growth rates corresponding to a shock propagating from a light to a heavy gas and vice versa. Good agreement with the Richtmyer formula, Eq. (6), was obtained for the light-to-heavy case; however, better agreement in the heavy-to-light case than that given by Eq. (6) was obtained by averaging the pre- and post-shock amplitudes,

$$
\frac{\mathrm{d} a}{\mathrm{~d} t}=k \Delta u A^{+} \frac{a_{0}^{+}+a_{0}^{-}}{2} .
$$

Integrating gives

$$
a(t)=a_{0}^{+}+k \Delta u A^{+} \frac{a_{0}^{+}+a_{0}^{-}}{2} t .
$$

\section{The Fraley model}

Fraley ${ }^{45}$ presented an analytic solution to the linearized perturbation equations in the case of a reflected shock wave. The complete set of linearized, compressible perturbation equations was first solved numerically by Richtmyer. ${ }^{1}$ Fraley reconsidered the perturbation equations for a single-mode initial perturbation and solved them using Laplace transform techniques in the time domain. For weak shocks the solution is (see Ref. 4)

$$
\frac{\mathrm{d} a}{\mathrm{~d} t}=k \Delta u a_{0}^{-}\left[A^{-}+\epsilon \frac{F\left(c, A^{-}\right)}{\gamma_{1}}\right],
$$

where $\epsilon=1-p^{-} / p^{+}$is the shock strength,

$$
\begin{aligned}
F\left(c, A^{-}\right) \equiv & \left\{\frac{(c-1)^{2}}{2}-\frac{1+A^{-}}{1-A^{-}}-c\right. \\
& \left.+\frac{1}{c}\left[\frac{\left(1+A^{-}\right)^{2}}{1-A^{-}}+\left(1-A^{-}\right) c^{2}\right]\right\} \frac{1-A^{-}}{c+1},
\end{aligned}
$$

$c \equiv \sqrt{\left(1+A^{-}\right) \gamma_{2} /\left(1-A^{-}\right) \gamma_{1}}$, and $\gamma_{1}$ and $\gamma_{2}$ are the adiabatic exponents of the ideal gases to the left and right of the interface, respectively. Integrating Eq. (10) gives

$$
a(t)=a_{0}^{+}+k \Delta u\left[A^{-}+\epsilon \frac{F\left(c, A^{-}\right)}{\gamma_{1}}\right] a_{0}^{-} t .
$$

The Fraley solution was first recognized by Mikaelian ${ }^{46}$ as the most accurate solution for the initial perturbation growth.

\section{The Vandenboomgaerde-Mügler-Gauthier model}

Vandenboomgaerde, Mügler, and Gauthier ${ }^{47}$ modified the Richtmyer model by replacing the impulsive acceleration, post-shock Atwood number, and post-shock amplitude with linearly time-varying values from pre- to post-shock quantities, obtaining

$$
\frac{\mathrm{d} a}{\mathrm{~d} t}=k \Delta u\left[\frac{A^{+} a_{0}^{+}+A^{-} a_{0}^{-}}{2}-\frac{\left(A^{+}-A^{-}\right)\left(a_{0}^{+}-a_{0}^{-}\right)}{6}\right] .
$$

In applications of this formula, the second term on the right is typically very small compared to the first term and is neglected. Integrating Eq. (12) gives

$$
a(t)=a_{0}^{-}+k \Delta u\left[\frac{A^{+} a_{0}^{+}+A^{-} a_{0}^{-}}{2}-\frac{\left(A^{+}-A^{-}\right)\left(a_{0}^{+}-a_{0}^{-}\right)}{6}\right] t .
$$

\section{B. Perturbation models}

Models based on the asymptotic expansion of the linear perturbation equations are briefly reviewed here. These models generate asymptotic series with limited radii of convergence: the convergence can be improved using Padé approximants.

\section{The Zhang-Sohn model}

Zhang and Sohn ${ }^{48}$ developed a model for the growth of a two-dimensional Richtmyer-Meshkov unstable interface from early to late times in the case of a reflected shock (lightto-heavy transition). The dynamics of the interface are modeled using the linear, compressible flow equations for early times and the nonlinear, incompressible flow equations for later times. Let $y=\eta(x, t)$ denote the initial perturbation and let $\phi_{r}(x, y, t)$ denote the velocity potentials of the inviscid, irrotational fluids $r=1$ and 2 . The potentials satisfy the Laplace equation $\nabla^{2} \phi_{r}=0$ with interfacial kinematic boundary condition $\partial \eta /\left.\partial t\right|_{y=\eta}=\left.\left(\partial \phi_{r} / \partial x\right)(\partial \eta / \partial x)\right|_{y=\eta}-\partial \phi_{r} /\left.\partial y\right|_{y=\eta}$, together with the Bernoulli equation $\rho_{1}\left(\partial \phi_{1} / \partial t-\left|\nabla \phi_{1}\right|^{2} / 2\right)$ $=\rho_{2}\left(\partial \phi_{2} / \partial t-\left|\nabla \phi_{2}\right|^{2} / 2\right)+\left(\rho_{1}-\rho_{2}\right) \Delta u \delta(t) \eta$ at $y=\eta$, where $\boldsymbol{\nabla}$ $=(\partial / \partial x, \partial / \partial y)$. The initial conditions are $\eta(x, 0)=a_{0}^{+} \cos (k x)$ and $\mathrm{d} \eta /\left.\mathrm{d} t\right|_{t=0}=a_{0}^{+} \sigma \cos (k x)$, where $\sigma \equiv k A^{+} \Delta u=v_{0} / a_{0}^{+}$. 
Substituting the perturbation expansions $\eta(x, t)$ $=\sum_{n=1}^{\infty} \eta^{(n)}(x, t)$ and $\phi_{r}(x, y, t)=\sum_{n=0}^{\infty} \phi_{r}^{(n)}(x, y, t)$ into the equations, collecting terms of the same order, and solving the ordinary differential equations in time yields $\eta^{(1)}(x, t)$ $=a_{0}^{+}(1+\sigma t) \cos (k x), \quad \eta^{(2)}(x, t)=\frac{1}{2} k\left(\sigma a_{0}^{+}\right)^{2} A^{+} t^{2} \cos (2 k x)$, and $\quad \eta^{(3)}(x, t)=-\frac{1}{24} k^{2}\left(a_{0}^{+}\right)^{3} \sigma\left\{\left[4\left(A^{+}\right)^{2}+1\right] \sigma^{2} t^{3}+3 \sigma t^{2}\right.$ $+6 t\} \cos (k x)+\frac{1}{8} k^{2}\left(a_{0}^{+}\right)^{3} \sigma\left\{\left[4\left(A^{+}\right)^{2}-1\right] \sigma^{2} t^{3}-3 \sigma t^{2}\right\} \cos (3 k x)$ to third order. The series approximation can be evaluated at the spike and bubble tip locations $(x=0$ and at $x=\pi / k$, respectively) to yield the amplitude $a(t)=[\eta(0, t)-\eta(\pi / k, t)] / 2$. This formulation gives an independent series for the spike and bubble, which can be used to evaluate separate bubble and spike amplitude models. The amplitude growth is

$$
\frac{\mathrm{d} a}{\mathrm{~d} t}=\left\{1-k^{2} v_{0} a_{0}^{+} t+\left[\left(A^{+}\right)^{2}-\frac{1}{2}\right]\left(k v_{0} t\right)^{2}\right\} v_{0},
$$

where $v_{0}$ is the Richtmyer velocity. Integrating gives

$$
a(t)=a_{0}^{+}+\left\{1-\frac{k^{2} v_{0} a_{0}^{+}}{2} t+\frac{1}{3}\left[\left(A^{+}\right)^{2}-\frac{1}{2}\right]\left(k v_{0} t\right)^{2}\right\} v_{0} t .
$$

Unfortunately, the range of validity of this finite Taylor series approximation is limited. Hence, Padé approximations (see the discussion of the Vandenboomgaerde et al. model below) are used to extend the approximation into the nonlinear regime,

$$
\frac{\mathrm{d} a}{\mathrm{~d} t}=\frac{v_{0}}{1+k^{2} v_{0} a_{0}^{+} t+\max \left[0,\left(k a_{0}^{+}\right)^{2}-\left(A^{+}\right)^{2}+\frac{1}{2}\right]\left(k v_{0} t\right)^{2}} .
$$

Integrating Eq. (16) gives

$$
\begin{aligned}
a(t)= & a_{0}^{+}+\frac{2}{k \sqrt{4 \max \left[0,\left(k a_{0}^{+}\right)^{2}-\left(A^{+}\right)^{2}+\frac{1}{2}\right]-\left(k a_{0}^{+}\right)^{2}}} \\
& \times \tan ^{-1}\left[\frac{k a_{0}^{+}+2 \max \left[0,\left(k a_{0}^{+}\right)^{2}-\left(A^{+}\right)^{2}+\frac{1}{2}\right] k v_{0} t}{\sqrt{4 \max \left[0,\left(k a_{0}^{+}\right)^{2}-\left(A^{+}\right)^{2}+\frac{1}{2}\right]-\left(k a_{0}^{+}\right)^{2}}}\right] .
\end{aligned}
$$

The choice of the Padé approximant $P_{2}^{0}$ for $\left(k a_{0}^{+}\right)^{2} \geq\left(A^{+}\right)^{2}$ $-1 / 2$ and $P_{1}^{0}$ for $\left(k a_{0}^{+}\right)^{2} \leq\left(A^{+}\right)^{2}-1 / 2$ matches the experimental late-time growth. Note that $v_{0}$ in Eq. (16) can be replaced by $v_{\text {lin }}(t),{ }^{48}$ the numerically determined linear growth rate obtained by solving the full set of compressible perturbation equations. ${ }^{1}$ In this case, Eq. (16) must be integrated numerically to obtain $a(t)$. The Zhang-Sohn model does not predict the generally accepted $1 / t$ growth at late times.

\section{The Vandenboomgaerde-Gauthier-Mügler model}

Vandenboomgaerde, Gauthier, and Mügler ${ }^{49}$ proposed a simplified version of the Zhang-Sohn perturbation expansion. First, choose $\sigma \equiv(k \Delta u / 2)\left[A^{+}+A^{-} /(1\right.$ $\left.\left.-\Delta u / u_{\text {shock }}\right)\right]$ such that $a_{0}^{-} \sigma$ gives the right side of Eq. (12), where $u_{\text {shock }}$ is the shock velocity. Noting that an accurate perturbation series can be obtained by retaining only the secular terms (i.e., the terms with the largest unbounded part), only the largest power from each term of the Zhang-Sohn solution is retained to third order, $\eta^{(1)}(x, t)$ $=a_{0}^{+}(1+\sigma t) \cos (k x), \quad \eta^{(2)}(x, t)=\frac{1}{2}\left(a_{0}^{+} \sigma t\right)^{2} k A^{+} \cos (2 k x)$, and $\eta^{(3)}(x, t)=-\frac{1}{8}\left(k a_{0}^{+}\right)^{2}(\sigma t)^{3}\left\{\frac{1}{3}\left[4\left(A^{+}\right)^{2}+1\right] \cos (k x)-\left[4\left(A^{+}\right)^{2}\right.\right.$ $-1] \cos (3 k x)\} a_{0}^{+}$. Such an approximation is usually valid for large times, and the first two terms of this series are identical to the Zhang-Sohn series, so that very good agreement is expected between the predictions of this model and the Zhang-Sohn model at small times. An advantage of this perturbation method is that higher-order terms can be easily computed.

As only the high-order terms in the series are retained, the determination of the coefficients shifts from integrating in time to solving an algebraic system. The growth rate is given by the $2 N$ th-degree polynomial

$$
\frac{\mathrm{d} a}{\mathrm{~d} t}=a_{0}^{+} \sigma \sum_{n=0}^{N}(2 n+1)\left(k a_{0}^{+} \sigma t\right)^{2 n} P_{2 n+1}\left(A^{+}\right),
$$

where $P_{2 n+1}$ are the series approximation in $A^{+}$(not shown). Integrating gives

$$
a(t)=a_{0}^{+}+\frac{1}{k} \sum_{n=0}^{N}\left(k a_{0}^{+} \sigma t\right)^{2 n+1} P_{2 n+1}\left(A^{+}\right),
$$

where $N=4$ and 5 correspond to the ninth- and eleventhdegree cases, respectively. The $P_{6}^{4}$ Padé approximant to $\mathrm{d} a / \mathrm{d} t$ is

$$
\frac{\mathrm{d} a}{\mathrm{~d} t}=\frac{\sum_{p=0}^{4} A_{p}\left(k a_{0}^{+} \sigma t\right)^{p}}{\sum_{q=0}^{6} B_{q}\left(k a_{0}^{+} \sigma t\right)^{q}}
$$

where the Padé coefficients $\left\{A_{p}\right\}$ and $\left\{B_{q}\right\}$ are not given here.

\section{The Matsuoka-Nishihara-Fukuda model}

Matsuoka, Nishihara, and Fukuda ${ }^{50}$ proposed a new formulation of the Zhang-Sohn kinematic boundary condition to account for interface stretching: this boundary condition was replaced by $\overline{\mathbf{u}} \cdot \mathbf{n}=\left[\left(\rho_{1} \nabla \phi_{1}+\rho_{2} \nabla \phi_{2}\right) \cdot \mathbf{n}\right] /\left(\rho_{1}+\rho_{2}\right)$ and $\overline{\mathbf{u}} \cdot \mathbf{S}$ $=\left[\left(\rho_{1} \nabla \phi_{1}+\rho_{2} \nabla \phi_{2}\right) \cdot \mathbf{s}\right] /\left(\rho_{1}+\rho_{2}\right)$ evaluated on the interface, where $\overline{\mathbf{u}} \equiv\left(\rho_{1} \mathbf{u}_{1}+\rho_{2} \mathbf{u}_{2}\right) /\left(\rho_{1}+\rho_{2}\right)$ is the density-weighted mean velocity, $\mathbf{n}$ is the unit normal vector to the interface, and $\mathbf{s}$ is the unit tangent vector to the interface. The perturbation expansion yields different expansions for the bubble and spike amplitude. When the first three terms are considered, the bubble and spike velocities are

$$
\begin{aligned}
\frac{\partial a_{b, s}}{\partial \tau}= & {\left[\mp\left(A^{+}\right)^{2} \pm \frac{1}{2}\right] \tau^{2}+\left[\mp 2\left(A^{+}\right)^{2} k a_{0}^{+}+A^{+} \pm k a_{0}^{+}\right] \tau } \\
& +\left[\mp 2\left(A^{+}\right)^{2} \pm \frac{3}{2}\right]\left(k a_{0}^{+}\right)^{2}+\frac{k a_{0}^{+} A^{+}}{2} \mp 1
\end{aligned}
$$

where $\tau$ is the rescaled time [Eq. (5)] and the upper (+ or - ) and lower $(-$ or +$)$ sign in \pm or $\mp$ denotes the bubble and spike, respectively. The asymptotic series can be improved by considering $P_{2}^{0}$ Padé approximants,

$$
\frac{\partial a_{b, s}}{\partial \tau}=\frac{f_{ \pm}^{3}}{\left\{f_{ \pm}\left[\left(A^{+}\right)^{2}-\frac{1}{2}\right]+g_{ \pm}^{2}\right\} \tau^{2}-f_{ \pm} g_{ \pm} \tau+f_{ \pm}^{2}},
$$

where $f_{ \pm} \equiv \mp\left[2\left(A^{+}\right)^{2}-3 / 2\right]\left(k a_{0}^{+}\right)^{2}+A^{+} k a_{0}^{+} / 2 \mp 1$ and $g_{ \pm}$ $\equiv \mp 2\left(A^{+}\right)^{2} k a_{0}^{+}+A^{+} \pm k a_{0}^{+}$. Integrating gives 


$$
\begin{aligned}
a_{b, s}(\tau)= & a_{b, s}(0)+\frac{2 f_{ \pm}^{2}}{\sqrt{4 f_{ \pm}\left[\left(A^{+}\right)^{2}-\frac{1}{2}\right]+3 g_{ \pm}^{2}}} \\
& \times \tan ^{-1}\left\{\frac{2\left\{f_{ \pm}\left[\left(A^{+}\right)^{2}-\frac{1}{2}\right]+g_{ \pm}^{2}\right\} \tau-f_{ \pm} g_{ \pm}}{f_{ \pm} \sqrt{4 f_{ \pm}\left[\left(A^{+}\right)^{2}-\frac{1}{2}\right]+3 g_{ \pm}^{2}}}\right\} .
\end{aligned}
$$

\section{The Sadot et al. model}

The empirical Sadot et al. ${ }^{51}$ model for the RichtmyerMeshkov instability is based on fits to experimental data and on asymptotic growth laws. This model was extensively tested against experimental data and appears to be valid over $\mathrm{Ma}=1.3-3.5$ in air and $\mathrm{SF}_{6}$. The model provides a single formula that captures the initial linear growth, and the later nonlinear growth of the bubble and spike,

$$
\frac{\mathrm{d} a_{b, s}}{\mathrm{~d} t}=\frac{\left(1+k v_{0} t\right) v_{0}}{1+\left(1 \pm A^{+}\right) k v_{0} t+\frac{1 \pm A^{+}}{1+A^{+}} \frac{\left(k v_{0} t\right)^{2}}{2 \pi C}}
$$

[the upper (+) and lower (-) sign in \pm denote the bubble and spike, respectively] where $C=1 /(3 \pi)$ for $A^{+} \geq 0.5$. In the limit $A^{+} \rightarrow 0, C=1 /(2 \pi)$. Integrating Eq. (24) gives

$$
\begin{aligned}
a_{b, s}(t)= & a_{b, s}(0)+\frac{2-2 \pi C\left(1+A^{+}\right)}{k \sqrt{\frac{2}{\pi C} \frac{1 \pm A^{+}}{1+A^{+}}-\left(1 \pm A^{+}\right)^{2}}} \\
& \times \tan ^{-1}\left[\frac{1 \pm A^{+}+\frac{1 \pm A^{+}}{1+A^{+}} \frac{k v_{0} t}{\pi C}}{\sqrt{\frac{2}{\pi C} \frac{1 \pm A^{+}}{1+A^{+}}-\left(1 \pm A^{+}\right)^{2}}}\right] \\
& +\frac{\pi C}{k} \frac{1+A^{+}}{1 \pm A^{+}} \ln \left[1+\left(1 \pm A^{+}\right) k v_{0} t\right. \\
& \left.+\frac{1 \pm A^{+}}{1+A^{+}} \frac{\left(k v_{0} t\right)^{2}}{2 \pi C}\right] .
\end{aligned}
$$

This model is consistent with $1 / t$ asymptotic growth at late times.

\section{Potential flow models}

Potential flow models describe the bubble and spike velocity through the late-time, nonlinear regime. Layzer ${ }^{52} \mathrm{de}-$ veloped the first potential flow model to describe the Rayleigh-Taylor instability, which was subsequently extended to the Richtmyer-Meshkov instability by others. These models predict that the bubble velocity approaches zero asymptotically in the Richtmyer-Meshkov instability.

\section{The Goncharov model}

Goncharov $^{53}$ extended the two-dimensional Layzer model to the $A^{+} \neq 1$ case using a parabolic expansion $\eta(t)$ $=h(t)+\xi(t) x^{2}$ near the bubble and spike tips and initial perturbations $\phi_{1}(x, y, t)=a_{1}(t) \cos (k x) e^{-k[y-h(t)]}$ and $\phi_{2}(x, y, t)$ $=b_{1}(t) \cos (k x) e^{k[y-h(t)]}+b_{2}(t) y$. This ansatz requires the solution of five ordinary differential equations for the functions
TABLE II. The interface velocity $\Delta u$ and Richtmyer velocity $v_{0}$ from the ninth-order simulation and experiment. The pre- and post-shock values of

\begin{tabular}{|c|c|c|c|c|}
\hline & \multicolumn{2}{|c|}{ Simulation value } & \multicolumn{2}{|c|}{ Experimental value } \\
\hline$\Delta u(\mathrm{~cm} / \mathrm{s})$ & \multicolumn{2}{|c|}{6731} & \multicolumn{2}{|c|}{6060} \\
\hline$v_{0}(\mathrm{~cm} / \mathrm{s})$ & \multicolumn{2}{|c|}{702} & \multicolumn{2}{|c|}{628} \\
\hline \multirow[t]{2}{*}{$\underline{\eta_{\text {comp }}}$} & \multicolumn{2}{|c|}{0.8071} & \multicolumn{2}{|c|}{-} \\
\hline & Pre-shock & Post-shock & Pre-shock & Post-shock \\
\hline$a_{0}(\mathrm{~cm})$ & 0.2 & 0.162 & 0.183 & 0.157 \\
\hline$A$ & 0.6053 & 0.6045 & 0.604 & 0.625 \\
\hline$\delta(\mathrm{cm})$ & 0.5 & 0.4035 & 0.5 & - \\
\hline$\psi$ & 1.182 & 1.145 & 1.17 & 1.08 \\
\hline
\end{tabular}
the perturbation amplitude $a_{0}$, Atwood number $A$ (obtained from onedimensional shock refraction theory), the diffuse interface thickness $\delta$ [the pre-shock experimental value was taken from Jones and Jacobs (Ref. 41), which is the same as that in the Collins and Jacobs experiment], and growth reduction factor $\psi$ [obtained by solving the eigenvalue Eq. (33)] are also compared to the values reported by Collins and Jacobs (Ref. 15).

$a_{1}(t), b_{1}(t), b_{2}(t), h(t)$, and $\xi(t)$. The asymptotic bubble velocity for the Richtmyer-Meshkov instability is obtained by taking the $t \rightarrow \infty$ limit of the result,

$$
v_{b}(t)=\frac{3+A^{+}}{3\left(1+A^{+}\right) k t} \text {. }
$$

\section{The Sohn models}

Sohn ${ }^{54}$ also extended the Layzer model to fluids with arbitrary density ratios. The approach differs from that of Goncharov in the use of a simpler form for the initial potentials, $\quad \phi_{1}(x, y, t)=-a(t) \cos (k x) e^{-k y} \quad$ and $\quad \phi_{2}(x, y, t)$ $=a(t) \cos (k x) e^{-k y}$, leading to a simplified system of three ordinary differential equations for the functions $a(t), h(t)$, and $\xi(t)$. The Richtmyer-Meshkov asymptotic bubble velocity is

$$
v_{b}(t)=\frac{2}{\left(2+A^{+}\right) k t} .
$$

The ratio of Eq. (26) and Eq. (27), $\left(3+A^{+}\right)\left(2+A^{+}\right) /[6(1$ $\left.+A^{+}\right)$], quantifies the difference between the Sohn and Goncharov models. In the present case with $A^{+}=0.6045$ (see Table II) the ratio indicates that the predictions are within $2.5 \%$ of one another.

Sohn ${ }^{55,57}$ also extended the Zufiria model ${ }^{56}$ to arbitrary Atwood numbers. In the Zufiria model, originally developed to model bubbles of different size, the velocity potential is obtained by approximating the bubble as a point source. The bubble velocity is

$$
v_{b}(t)=\left[\frac{3+A^{+}}{3\left(1+A^{+}\right)}-\frac{1}{q}+\frac{2 A^{+}}{3\left(1+A^{+}\right) q^{2}}\right] \frac{1}{k t},
$$

where $q=q\left(A^{+}\right)$is the root of the cubic polynomial (3 $\left.-A^{+}\right) q^{3}-\left(21+9 A^{+}\right) q^{2}+\left(3+15 A^{+}\right) q-4 A^{+}=0$. Comparing the Zufiria model [Eq. (28)] with the Goncharov model [Eq. (26)] shows that the former contains two additional terms, resulting in a predicted asymptotic velocity $\approx 12.5 \%$ smaller for the present value $A^{+}=0.6045$. 


\section{The Mikaelian model for the bubble amplitude}

Mikaelian ${ }^{58}$ obtained an analytic expression for the transition of the bubble amplitude from the linear to the nonlinear regime by combining the Richtmyer initial growth [Eq. (6)] with the Goncharov late-time bubble growth for arbitrary Atwood numbers [Eq. (26)]. Mikaelian used a technique attributed by Layzer ${ }^{52}$ to Fermi for combining the initial and asymptotic bubble velocities. However, unlike the Fermi model, in which the transition between the linear and asymptotic velocity occurs when the two velocities are equal, Mikaelian proposed a transition between these velocities at an earlier time corresponding to $a_{b}=1 /(3 k)$, resulting in decreasing the bubble amplitude in accord with numerical and experimental observations. For times $t<t^{*} \equiv\left[1 /\left(3 k a_{0}^{+}\right)\right.$ $-1] /\left(k A^{+} \Delta u\right)$, the amplitude is given by Eq. (6), so that $a_{b}\left(t^{*}\right)=1 /(3 k)$. The bubble amplitude is

$$
a_{b}(t)=\frac{1}{3 k}\left\{1+\frac{3+A^{+}}{1+A^{+}} \ln \left[1+\frac{3 k v_{0}\left(1+A^{+}\right)}{3+A^{+}}\left(t-t^{*}\right)\right]\right\}
$$

for times $t>t^{*}$, so that $v_{b}$ agrees with Eq. (26) in the limit of large times.

\section{COMPARISON OF SIMULATION AMPLITUDES TO EXPERIMENTAL DATA AND TO MODEL PREDICTIONS}

The perturbation amplitude growth from the fifth- and ninth-order simulations is compared here to the experimental data of Collins and Jacobs, ${ }^{15}$ and to the predictions of the models in Sec. III. First, the method used to determine the amplitudes from the simulations is presented. Next, these amplitudes are compared to the experimental data points. The ninth-order amplitude is then compared to the predictions of impulsive and nonlinear perturbation models, with the model parameters computed self-consistently from the simulation. The bubble and spike amplitudes are also compared with the predictions of growth models, and with the asymptotic bubble velocities predicted by potential flow models. A local and a global metric is used to quantify the differences between the various predictions and between the simulation and experimental data-an average fractional deviation and the ratio of the model predictions to the ninthorder amplitude, respectively. Prior to differentiating $a(t)$, the numerical amplitude was smoothed using a standard fivepoint moving average ${ }^{59}$ to reduce oscillations otherwise present.

\section{A. Determination of the bubble, spike, and mixing layer amplitudes}

Consider the mole fraction

$$
X(x, y, t)=\frac{m(x, y, t) M_{1}}{[1-m(x, y, t)] M_{2}+m(x, y, t) M_{1}},
$$

where $M_{1}$ and $M_{2}$ are the molecular weights of the air(acetone) and $\mathrm{SF}_{6}$, respectively, and $m$ is the mass fraction of $\mathrm{SF}_{6}$. Spatially averaging $X(x, y, t)$ in the spanwise direction gives

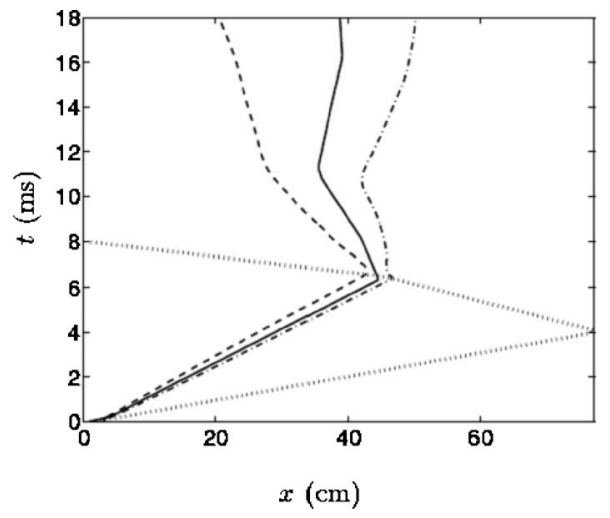

FIG. 5. The $x$ - $t$ diagram showing the position of the interface $\ell_{\text {int }}(t)$ (solid line), shock (dotted line), and bubble and spike tip locations $\ell_{b}(t)$ and $\ell_{s}(t)$ (dash-dot and dashed lines, respectively) from the ninth-order simulation. The horizontal distance between the spike and bubble tip location is the mixing layer width $h(t)$.

$$
\bar{X}(x, t)=\frac{1}{L_{y}} \int_{0}^{L_{y}} X(x, y, t) \mathrm{d} y,
$$

where $L_{y}$ is the spanwise domain width. The bubble and spike tip locations, $\ell_{b}(t)$ and $\ell_{s}(t)$, are then defined as the $x$ position where $\bar{X} \leq 1-\epsilon$ and $\bar{X} \geq \epsilon$, respectively, with $\epsilon$ $=0.01$ in the present investigation. ${ }^{60}$ The width is given by $h(t)=\ell_{b}(t)-\ell_{s}(t)$. This measure of the width was chosen, rather than using the distance between the lines $x=\lambda / 2$ and $x=\lambda$, in order to also examine the evolution of the postreshock width when the interface becomes multivalued and disordered. To obtain the bubble and spike amplitudes separately, a simulation without an initial perturbation, but otherwise identical to the Richtmyer-Meshkov instability simulation, was performed to obtain the interface position $\ell_{\mathrm{int}}$, so that the bubble and spike amplitudes are determined separately by $a_{b}(t) \equiv \ell_{b}(t)-\ell_{\text {int }}(t) \quad$ and $\quad a_{s}(t) \equiv \ell_{\text {int }}(t)-\ell_{s}(t)$, respectively.

The interface and shock locations from the ninth-order simulation are exhibited in the $x-t$ diagram of Fig. 5. The horizontal distance between the spike and bubble tips represents the width $h(t)$ (see Fig. 4 for a schematic of the amplitudes). The shock reflects from the end wall at $\approx 4 \mathrm{~ms}$ and reshock occurs at $t \approx 6.4 \mathrm{~ms}$ when the shock wave refracts at the evolving interface, generating a transmitted shock in the air(acetone) and a reflected rarefaction in the $\mathrm{SF}_{6}$ (not shown in the $x$ - $t$ diagram). The transmitted shock following reshock moves faster than the incident shock, as indicated by the change in slope, corresponding to a slow-fast refraction. ${ }^{61}$ Following reshock, the interface is compressed (as seen from the kink in the bubble and spike locations) and moves away from the end wall of the test section. Additionally, the amplitude grows more rapidly than prior to reshock: the increased growth is due to the additional vorticity deposited on the evolving interface during reshock. The reshock (inversion) process occurs over a time interval of $\approx 0.2-0.3 \mathrm{~ms}$.

The following conventions are used when comparing the numerical simulation data to the predictions of the models presented in Sec. III. First, Mikaelian ${ }^{62}$ extended the work of 

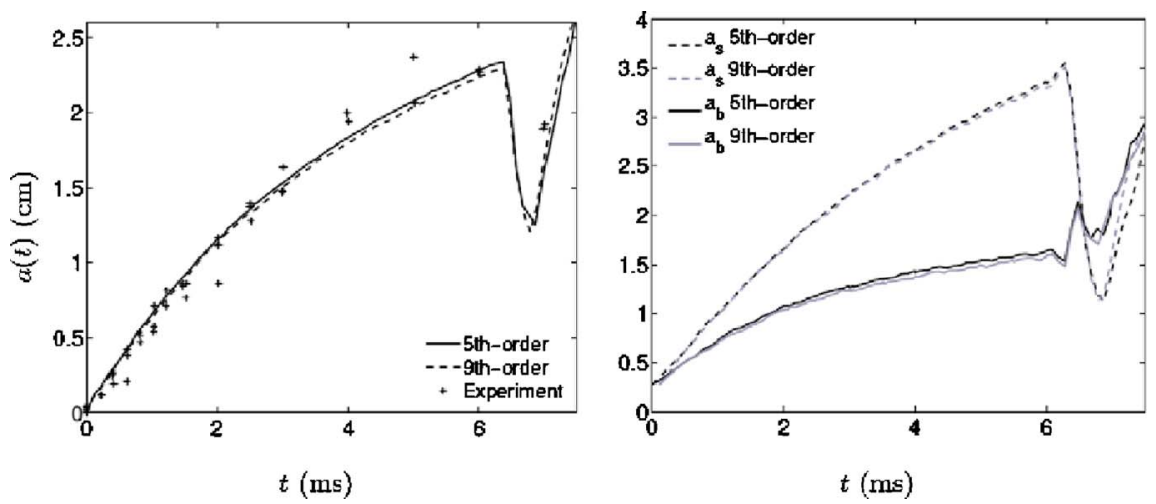

FIG. 6. Comparison of the amplitude obtained from the fifth- and ninthorder WENO simulation to the experimental data points from Collins and Jacobs (Ref. 15) (left). The bubble and spike amplitudes from the simulations, $a_{b}(t)$ and $a_{s}(t)$, respectively, are also shown (right).

Duff, Harlow, and Hirt ${ }^{63}$ considering the growth of diffuse interfaces in the Rayleigh-Taylor instability to the Richtmyer-Meshkov instability, obtaining

$$
\frac{\mathrm{d} a}{\mathrm{~d} t}=\frac{k \Delta u A^{+}}{\psi^{+}} a_{0}^{+},
$$

where $\psi^{+}$is the growth reduction factor (in general, a function of $a$ and $A^{+}$) satisfying the boundary value problem

$$
\frac{\mathrm{d}}{\mathrm{d} x}\left(\rho \frac{\mathrm{d} f}{\mathrm{~d} x}\right)=\left(\rho-\frac{\psi^{+}}{k A^{+}} \frac{\mathrm{d} \rho}{\mathrm{d} x}\right) k^{2} f
$$

where the eigenfunction satisfies $f \rightarrow 0$ as $x \rightarrow \pm \infty$. This equation was solved assuming a density profile of the form $\rho(x)=\bar{\rho}\left[1+A^{+} \tanh (x / \delta)\right]$ or $\rho(x)=\bar{\rho}\left[1+A^{+} \operatorname{erf}(x / \delta)\right]$, where $\bar{\rho}=\left(\rho_{1}+\rho_{2}\right) / 2$, so that the change in density occurs over a diffuse interface width $\delta$. Integrating Eq. (32) gives

$$
a(t)=\left(1+\frac{k \Delta u A^{+}}{\psi^{+}} t\right) a_{0}^{+} .
$$

Thus, in the comparisons of the simulation amplitude data to the predictions of the models shown later, the amplitude growth rates are rescaled to account for the diffuse interface by

$$
\frac{\mathrm{d} a}{\mathrm{~d} t} \rightarrow \frac{1}{\psi} \frac{\mathrm{d} a}{\mathrm{~d} t},
$$

which was also used by Collins and Jacobs ${ }^{15}$ to compare their amplitude data to growth model predictions. Here, $\psi$ was computed self-consistently by solving Eq. (33) using the numerical procedure described by Duff et al. ${ }^{63}$ and the initial error function density profile of Collins and Jacobs. The preand post-shock values of $\psi$ are shown in Table II, where the post-shock diffuse interface thickness was $\delta^{+}=\eta_{\text {comp }} \delta$ with $\eta_{\text {comp }}=1-\Delta u / u_{\text {shock }}$ the compression factor. The $\psi$ computed here differ slightly from the values reported by Collins and Jacobs due to slightly different input parameters in Eq. (33). The time and initial velocity are also rescaled according to (5), where $a_{0}^{+}$is determined by multiplying the pre-shock amplitude $a_{0}^{-}$by $\eta_{\text {comp. }}$. Reshock occurs at $\tau \lesssim 5$. In general, when a model predicts the growth rate, $\mathrm{d} a / \mathrm{d} t$, the amplitude $a(t)$ can be obtained by solving an initial value problem using a fourth-order Runge-Kutta scheme (for example).

Table II gives the parameters used in the amplitude growth model predictions, which are also compared to the experimental values (see Table I in Ref. 15). An $11 \%$ discrepancy exists between the interface velocity $\Delta u$ after the passage of the incident shock reported here and the experimental value inferred from Eq. (6), which was also used by Collins and Jacobs. A one-dimensional analysis assuming ideal gas behavior and a nonventilated shock tube gives $\Delta u$ $=6420 \mathrm{~cm} / \mathrm{s}$ while the measured speed is $\Delta u=6060 \mathrm{~cm} / \mathrm{s}$, which is attributed to the slots in the shock tube. ${ }^{15}$ The single-gamma limitation of the present simulations only accounts for the difference between the speed of $6731 \mathrm{~cm} / \mathrm{s}$ and the one-dimensional shock refraction theory value of $6420 \mathrm{~cm} / \mathrm{s}$.

\section{B. Comparison to experimental amplitude data}

The fifth- and ninth-order simulation amplitudes are compared to the experimental amplitude data in Fig. 6. The layer begins growing immediately following the initial shock passage. The initial rapid growth saturates at $\approx 4 \mathrm{~ms}$, and reshock occurs $\approx 2.4 \mathrm{~ms}$ later. During reshock, the amplitude is compressed by $\approx 1 \mathrm{~cm}$ and subsequently grows rapidly. The numerical and experimental data are in very good agreement up to the time at which the driver-based expansion present in the experiment (but not present in either the simulations or models) begins to expand the layer at $\approx 4.5$ $-5 \mathrm{~ms}$ (see Fig. 4 of Collins and Jacobs ${ }^{15}$ ). The subsequent discrepancy is due to the arrival of the driver-based expansion wave not modeled in the simulation, and to the difference in interface velocity between the simulation and experiment. The driver-based expansion decelerates the interface and, thus, delays the reshock to $t>8 \mathrm{~ms}$ in the experiment. For $t>3 \mathrm{~ms}$, the fifth-order amplitude is slightly larger than the ninth-order amplitude up to reshock. The instability evolution in the experiment is essentially two-dimensional prior to reshock (by virtue of the manner in which the initial perturbation was produced), and becomes three-dimensional following reshock, as the shock-interface interaction excites fluctuations in all spatial directions. Thus, it is reasonable to expect that a two-dimensional numerical simulation can reproduce the experimentally measured amplitude growth prior to reshock. However, three-dimensional simulations are necessary to more accurately estimate the mixing layer width evolution and other quantities following reshock.

The simulation bubble and spike amplitudes are also shown in Fig. 6: the amplitude asymmetry is due to the rela- 


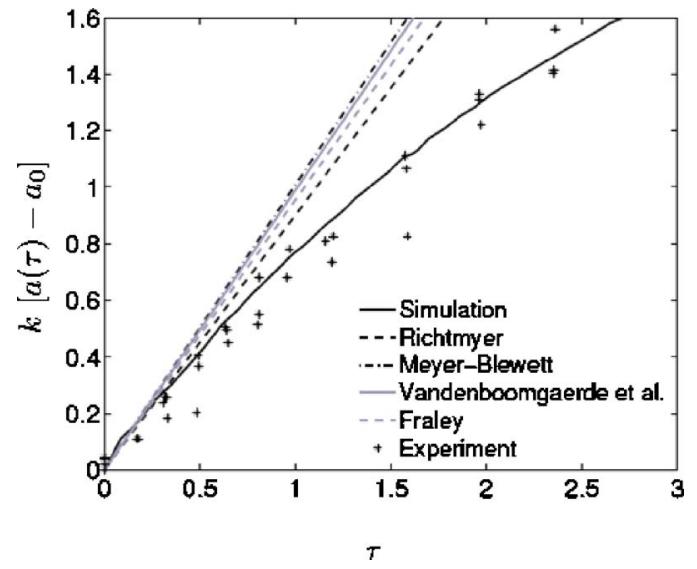

FIG. 7. The normalized amplitude $k\left[a(\tau)-a_{0}\right]$ versus $\tau$ from the ninth-order WENO simulation and the experimental data points, together with the predictions of the impulsive models. The Richtmyer model gives the smallest slope $(\mathrm{d} a / \mathrm{d} t=740.1 \mathrm{~cm} / \mathrm{s})$ as it uses the post-shock Atwood number and amplitude. The pre- and post-shock amplitudes are averaged in the MeyerBlewett model, resulting in the largest slope, $828.6 \mathrm{~cm} / \mathrm{s}$. The pre- and post-shock amplitudes are averaged by the pre- and post-shock Atwood numbers, respectively, in the Vandenboomgaerde model, which thus predicts a slightly smaller slope, $812.4 \mathrm{~cm} / \mathrm{s}$, than that predicted by the MeyerBlewett model, but larger than that predicted by the Richtmyer model. The Fraley model has an exact initial slope of $784.3 \mathrm{~cm} / \mathrm{s}$, intermediate between those predicted by the Meyer-Blewett and Vandenboomgaerde et al. models.

tively large Atwood number. Prior to reshock, the fifth-order bubble and spike amplitudes are slightly larger than the ninth-order amplitudes. Following reshock, the fifth-order bubble amplitude is slightly larger than the ninth-order amplitude, but the converse is true for the spike amplitude. To globally quantify the difference between the experiment and simulation, the average fractional deviation ${ }^{42}$

$$
\Delta=\frac{1}{N} \sum_{i=1}^{N} \frac{\left|a_{\text {sim }}\left(t_{i}\right)-a_{\exp }\left(t_{i}\right)\right|}{a_{\text {sim }}\left(t_{i}\right)}
$$

between the simulation data, $a_{\text {sim }}\left(t_{i}\right)$, and the experimental data points, $a_{\exp }\left(t_{i}\right)$, is computed at the times $t_{i}(i$ $=1,2, \ldots, N)$, where $N=51$ is the number of experimental data points and the simulation amplitudes are spline interpolated to the experimental times $t_{i}$. The results $\Delta_{\text {fifth }}=0.1$ and $\Delta_{\text {ninth }}=0.11$ indicate that both amplitude predictions are very similar. As a result, only the ninth-order amplitude is considered in the next sections.

\section{Comparison to the predictions of impulsive models}

Simulation and experimental data are compared here to the predictions of the impulsive models described in Sec. III A when they are corrected for the case of a diffuse interface by (35). The ninth-order amplitude is compared to the prediction of the Richtmyer ${ }^{1}$ model [Eq. (6)], the Meyer and Blewett ${ }^{44}$ model [Eq. (8)], the Fraley ${ }^{45}$ model [Eq. (10)], and the Vandenboomgaerde et $a .^{47}$ model [Eq. (12)] in Fig. 7. The normalized amplitude $k\left[a(\tau)-a_{0}\right]$ is shown versus the normalized time $\tau$ given by Eq. (5). The impulsive models all correctly capture the initial growth for $\tau<1$, although they slightly overestimate the amplitude. For $\tau>1$ (typically referred to as the nonlinear growth phase), nonlinear effects ensue and the models significantly overestimate the amplitude and its growth rate. The impulsive models predict a constant growth rate, whereas the simulation growth decreases steadily in time as in the experiment due to nonlinear saturation effects. Thus, it is difficult to determine which model agrees best with the data at early times. However, this is not the case for all initial conditions, as plotting the normalized growth rate as a function of the shock strength and comparing the predictions of the impulsive models to the Fraley (exact) solution would show. ${ }^{46}$ The impulsive models exhibit large deviations from the Fraley solution for strong shocks, indicating that they are no longer valid.

\section{Comparison to the predictions of perturbation models}

The numerical and experimental amplitude data are compared here to the predictions of the nonlinear perturbation models summarized in Sec. III B when they are corrected for the case of a diffuse interface by (35). The experimental and simulation data are compared to the perturbation series solutions of Zhang and $\mathrm{Sohn}^{48}$ [Eq. (14)], Vandenboomgaerde et al. ${ }^{49}$ [Eq. (18)] of degree 9 and 11, and Matsuoka et al. ${ }^{50}$ [Eq. (21)] in Fig. 8. Also shown in Fig. 8 is a comparison of the normalized growth rate, $k \mathrm{~d} a / \mathrm{d} \tau$, from the ninth-order simulation to the predictions of the nonlinear perturbation series models versus the normalized time $\tau$ : The perturbation series capture the initial growth, but quickly diverge. In particular, the eleventh-order Vandenboomgaerde $e t$ al. perturbation series has a smaller radius of convergence
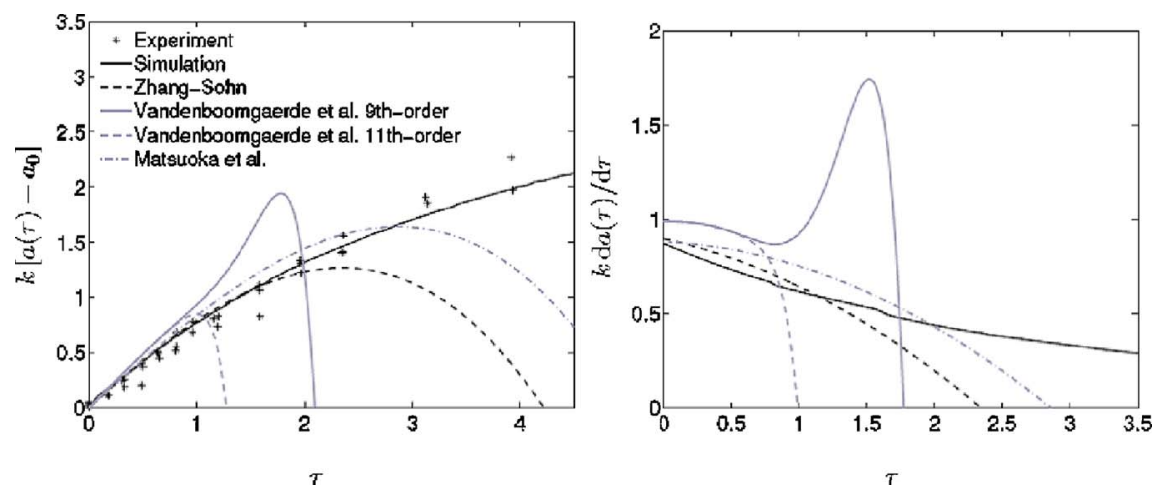

FIG. 8. The normalized amplitude $k\left[a(\tau)-a_{0}\right]$ versus $\tau$ from the ninthorder WENO simulation and the experimental data points, together with the predictions of the Zhang-Sohn, Vandenboomgaerde et al., and Matsuoka et al. perturbation series models (left). The normalized growth rates $k \mathrm{~d} a(\tau) / \mathrm{d} \tau$ versus $\tau$ from the ninthorder simulation, together with the corresponding growth rates from these models, are also shown (right). 

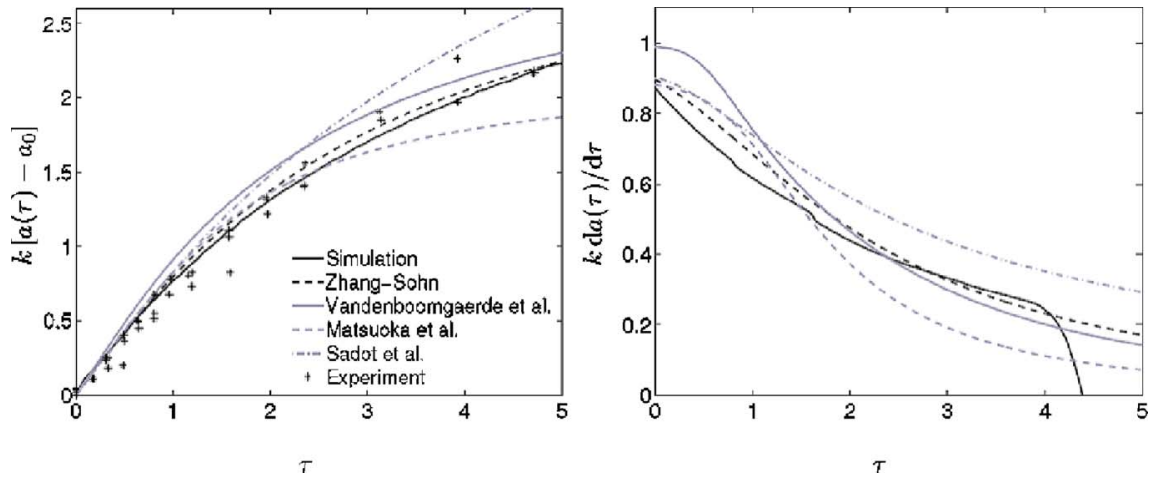

FIG. 9. The normalized amplitude $k\left[a(\tau)-a_{0}\right]$ versus $\tau$ from the ninthorder WENO simulation and the experimental data points, together with the predictions of the Zhang-Sohn, Vandenboomgaerde et al., and Matsuoka et al. models extended via Padé approximants, and the Sadot et al. model (left). The normalized growth rates $k \mathrm{~d} a(\tau) / \mathrm{d} \tau$ versus $\tau$ from the ninth-order simulation, together with the corresponding growth rates from these models, are also shown (right). than the ninth-order series: the series can be extended via Padé approximants to expand the radius of convergence.

The experimental and numerical data are compared to the predictions of the nonlinear models extended via Padé approximants in Fig. 9. Shown are the Zhang-Sohn $P_{2}^{0}$ Padé approximant [Eq. (16)], the Vandenboomgaerde et al. $P_{6}^{4}$ Padé approximant [Eq. (20)], and the Matsuoka et al. $P_{2}^{0}$ Padé approximant [Eq. (22)]. The prediction of the Sadot et $a l .{ }^{51}$ empirical model [Eq. (24)] is also shown. The ZhangSohn and Vandenboomgaerde et al. Padé model predictions agree well with the experimental data in the linear and nonlinear regimes. The Vandenboomgaerde et al. Padé approximant captures the correct behavior at early times $\tau<1$, but overestimates the amplitude at later times. The Zhang-Sohn Padé approximant underestimates the amplitude for $\tau<1$, but gives the correct behavior for later times. The Matsuoka et al. Padé approximant correctly predicts the amplitude at early times but underestimates the amplitude at late times. Also shown in Fig. 9 is the normalized growth rate, $k \mathrm{~d} a / \mathrm{d} \tau$, from the ninth-order simulation and the predictions of the nonlinear models. The nonlinear models considered here generally capture both the initial and late-time amplitude growth.

To determine which of the models gives the amplitude growth in best agreement with the simulation data, the ratios of the Zhang-Sohn (Padé), Vandenboomgaerde et al. (Padé), Matsuoka et al. (Padé), and Sadot et al. models to the simulation data, $\left[a_{\bmod }(\tau)-a_{0}\right] /\left[a_{\text {sim }}(\tau)-a_{0}\right]$, are presented in Fig.

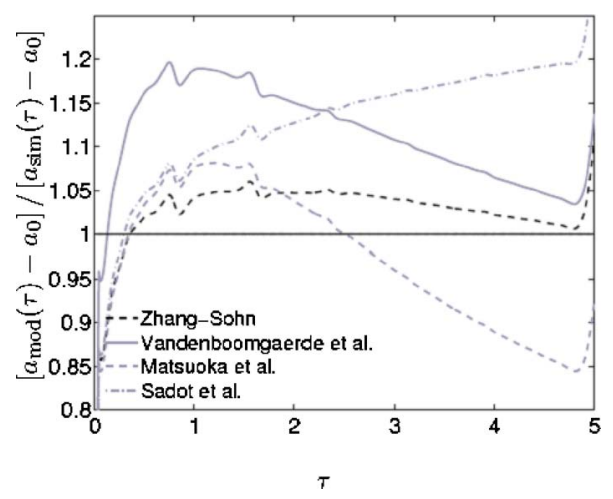

FIG. 10. The predictions of the Zhang-Sohn (Padé), Vandenboomgaerde et al. (Padé), Matsuoka et al. (Padé), and Sadot et al. models (with the initial perturbation amplitude subtracted out) divided by the corresponding ninthorder WENO simulation amplitude.
10. For $\tau \gtrsim 0.4$, the Zhang-Sohn Padé model is in best agreement with the simulation, overestimating the data by $<5 \%$ over the evolution time. Collins and Jacobs ${ }^{15}$ reported that their data are in best agreement with the amplitude predicted by the Sadot et al. model. Only the Sadot et al. model has the correct growth at late time, whereas the amplitudes from the other models attain a maximum between $\tau \approx 0.7$ and 1.6 and then rapidly decay up to reshock $\tau \lesssim 5$. To further support the conclusions of the present investigation, the average fractional deviation [Eq. (36)] is also computed between the simulation results and the model predictions, $\Delta_{\text {sim }}$, in Table III. The agreement between the simulation amplitudes and the experimental data, but the different conclusions regarding which amplitude growth model best agrees with the simulation and experimental data, motivates a study of the sensitivity of the model predictions to the model input parameters $\left(v_{0}, A^{+}, a_{0}^{+}\right.$, and $\left.\Delta u\right)$ presented in Sec. IV F.

TABLE III. Average fractional deviations between the simulation amplitude $a_{\text {sim }}(t)$ and the model prediction $a_{\text {mod }}(t)$ when experimental parameters are used $\left(\Delta_{\text {exp }}\right)$ and when simulation parameters are used $\left(\Delta_{\text {sim }}\right)$. Also shown are the average fractional deviations between the experimental amplitude $a_{\text {exp }}(t)$ and the model prediction $a_{\text {mod }}(t)$ when experimental parameters are used $\left(\Delta_{\text {exp }}^{e}\right)$ and when simulation parameters are used $\left(\Delta_{\text {sim }}^{e}\right)$.

\begin{tabular}{lcc}
\hline \hline & $\Delta_{\exp }(\%)$ & $\Delta_{\text {sim }}(\%)$ \\
\hline Zhang-Sohn Padé & 6.8 & 3.7 \\
Vandenboomgaerde et al. Padé & 1.9 & 12.3 \\
Matsuoka et al. Padé (all) & 12.0 & 9.8 \\
Matsuoka et al. Padé (bubble) & 26.4 & 22.7 \\
Matsuoka et al. Padé (spike) & 9.1 & 11.9 \\
Sadot et al. (all) & 4.5 & 14.9 \\
Sadot et al. (bubble) & 3.7 & 4.4 \\
Sadot et al. (spike) & 16.8 & 23.6 \\
Mikaelian (bubble) & 2.6 & 5.0 \\
\cline { 2 - 3 } & $\Delta_{\exp }^{e}(\%)$ & $\Delta_{\text {sim }}^{e}(\%)$ \\
\cline { 2 - 3 } Zhang-Sohn Padé & 9.9 & 11.9 \\
Vandenboomgaerde et al. Padé & 10.1 & 19.3 \\
Matsuoka et al. Padé & 11.7 & 14.4 \\
Sadot et al. & 8.4 & 15.7 \\
\hline \hline
\end{tabular}




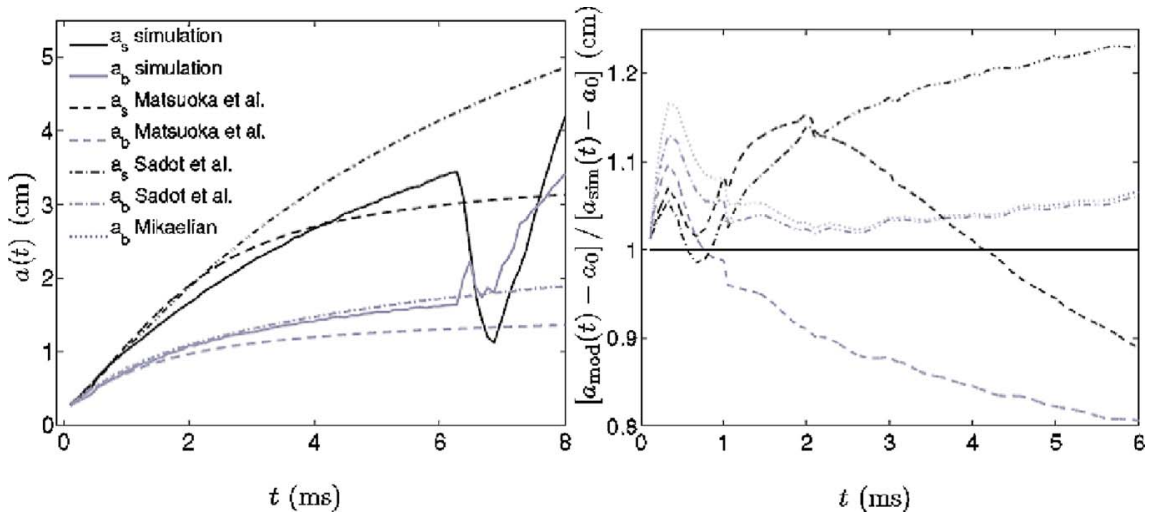

FIG. 11. Comparison of the bubble and spike amplitudes, $a_{b}(t)$ and $a_{s}(t)$, from the ninth-order WENO simulation with the predictions of the Matsuoka et al. (Padé) and Sadot et al. models, and with the Mikaelian model in the case of the bubble amplitude (left). Also shown are the ratios of the model predictions to the simulation bubble and spike amplitudes (with the initial perturbation amplitude subtracted out) (right).

\section{E. Comparison of bubble and spike amplitudes and velocities to the predictions of potential and perturbation models}

A comparison of the bubble and spike amplitudes from the ninth-order simulation to the Matsuoka et al. [Eq. (22)] and Sadot et al. [Eq. (24)] model predictions corrected for the case of a diffuse interface by (35) is shown in Fig. 11. The bubble amplitude prediction of the Mikaelian model [Eq. (29)] is also shown. The Matsuoka et al. model overestimates the bubble and spike amplitude. The Sadot et al. and Mikaelian models predict very good agreement for the bubble amplitude from the linear through the nonlinear regime. However, the Sadot et al. model overestimates the spike amplitude from the simulation. The ratio of the predicted bubble and spike amplitudes to the simulation results, respectively, is also shown in Fig. 11. The ratio shows an initial difference between the predictions of the Mikaelian and Sadot et al. models for the bubble amplitudes. However, this difference decreases, and by $3 \mathrm{~ms}$ the predictions of both models are nearly identical. For short times following the passage of the shock, the spike amplitude from the Matsuoka et al. model is in close agreement with the simulation amplitude. See Table III for the average fractional deviation [Eq. (36)] between the simulation results and the model predictions.

A comparison between the bubble and spike velocities from the simulation with the asymptotic bubble velocities predicted by the Goncharov model [Eq. (26)], the SohnLayzer model [Eq. (27)], and the Sohn-Zufiria model [Eq.
(28)], and the predictions of the bubble and spike velocities from the Matsuoka et al., Sadot et al., and Mikaelian models, are shown in Fig. 12. The asymptotic bubble velocities predicted by potential theory and the Sadot et al. and Mikaelian models are in very good agreement. The Sohn-Layzer and Goncharov models give nearly identical predictions. The bubble velocities predicted by the Sadot et al. model, the Mikaelian model, and the asymptotic velocity predicted by the Sohn-Zufiria model are all in very close agreement and are closest to the simulation bubble velocity. The Matsuoka et al. model underpredicts the late-time bubble velocity. The Sadot et al. and Matsuoka et al. model overpredicts and underpredicts the spike velocity, respectively.

\section{F. Comparison of amplitude model predictions using experimental and numerical model parameters}

The very good agreement between the numerical simulations and experiment shown in the previous section does not extend to the predictions of nonlinear growth models. When the amplitude model parameters, $v_{0}, A^{+}, a_{0}^{+}$, and $\Delta u$, are computed using the experimental data, the Sadot et al. model gives the best agreement with the experimental and simulation data; by contrast, when the parameters are computed using the simulation data, the Zhang-Sohn Padé model gives the best agreement. This is primarily due to the different initial growth velocity $v_{0}$ in the simulations and the experiment, and to the differences in $A^{+}, a_{0}^{+}$, and $\Delta u$. These observations and the single-gamma approximation used in
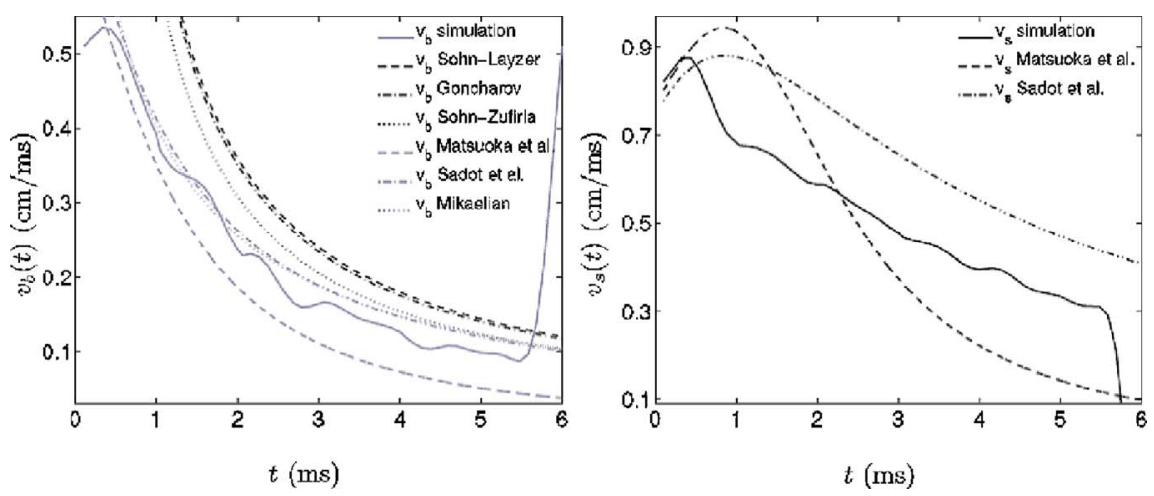

FIG. 12. The bubble velocity, $v_{b}(t)$, from the ninth-order WENO simulation with the asymptotic velocities predicted by the Sohn-Layzer, Goncharov, and Sohn-Zufiria models, and the velocities predicted by the Matsuoka et al., Sadot et al., and Mikaelian models (left). Also shown is the spike velocity, $v_{s}(t)$, and the velocities predicted by the Matsuoka et al. and Sadot et al. models (right). 

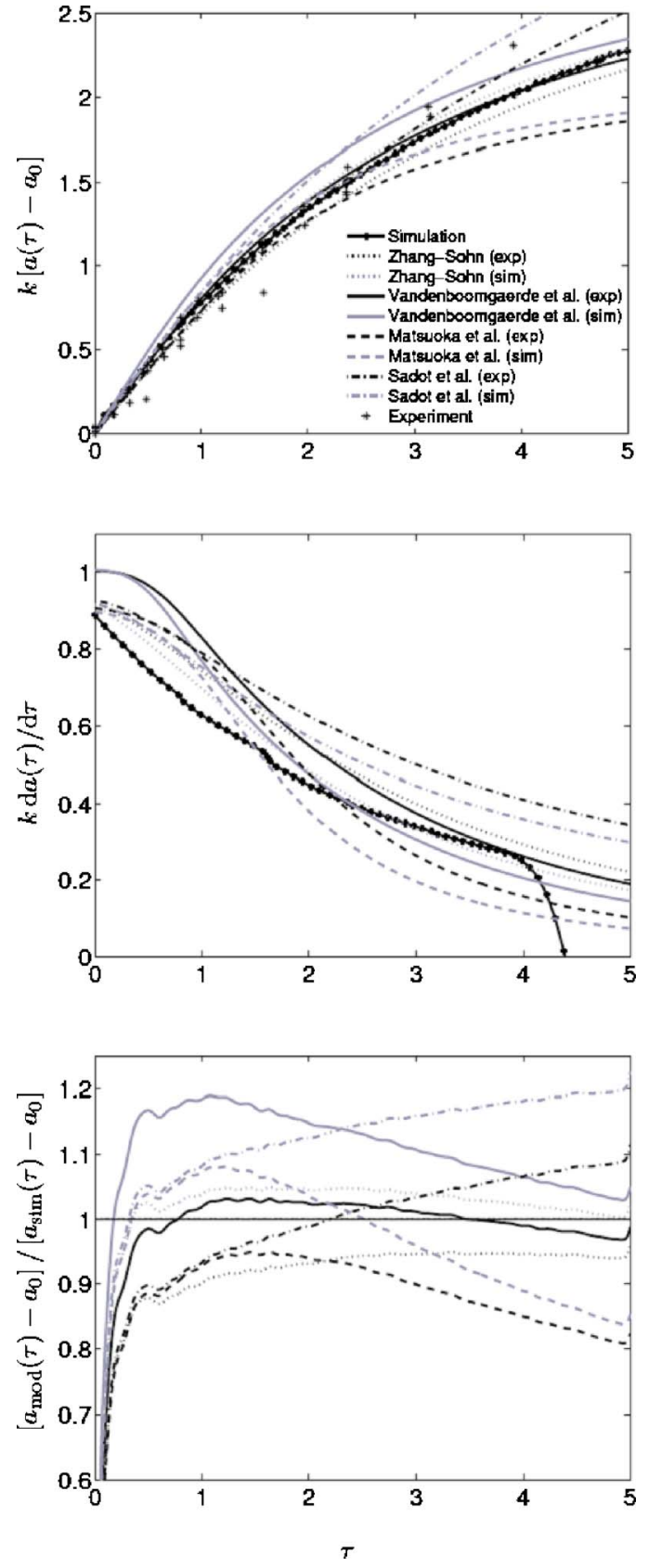

FIG. 13. The normalized amplitude predictions (with the initial perturbation amplitude subtracted out) of the nonlinear models using the experimental and simulation parameters $v_{0}, A^{+}, a_{0}^{+}$, and $\Delta u$ (as given in Table II), together with the experimental data points and the ninth-order WENO simulation data (top). The normalized amplitude growth rate predictions of the nonlinear models with the experimental and simulation parameters, together with the ninth-order simulation data, are also shown (middle). Also shown are the ratios of the model predictions to the simulation data (bottom).

the present simulations motivate a comparison of the nonlinear amplitude growth model predictions when the model parameters are computed using the experimental data (see Table II and Table I in Ref. 15) to those when the parameters are computed using the simulation data.

A comparison of the Zhang-Sohn Padé, Vandenboomgaerde et al. Padé, Matsuoka et al. Padé, and Sadot et al. model predictions computed using experimental and simulation values for $v_{0}, A^{+}, a_{0}^{+}$, and $\Delta u$ is presented in Fig. 13. The
Zhang-Sohn and Sadot et al. models are the most sensitive to changes in these model parameters. The predicted amplitudes obtained using the simulation parameters are in general larger than those obtained using the experimental parameters, due to the difference in the initial velocity of the interface: the simulation $v_{0}$ is larger than the corresponding experimental value. By contrast, the Vandenboomgaerde et al. model does not exhibit significant sensitivity to the variation of the experimental or simulation model parameters. When the experimental parameters are used, the Vandenboomgaerde et al. model prediction is in best agreement with the simulation; however, when the simulation parameters are used, the Zhang-Sohn model prediction is in best agreement with the simulation. The normalized amplitude growth rates predicted by the nonlinear growth models and the ratios of the model predictions to the ninth-order simulation data are also presented in Fig. 13.

A similar parametric sensitivity study is presented in Fig. 14 for the bubble and spike amplitudes and velocities, and their ratios to the corresponding simulation quantities. In general, when the experimental parameters are used, the predicted bubble and spike amplitudes are lower than those computed using the simulation parameters, resulting in better agreement with the simulation results. The velocities do not show a similar difference: in the case of the bubble velocity, the predictions obtained using the simulation and experimental parameters are in very close agreement.

The differences between the predictions of the models when the parameters are computed using the experimental and simulation data are further quantified using the average fractional deviation [Eq. (36)] between the simulation amplitude data $a_{\text {sim }}(t)$ and the model predictions $a_{\text {mod }}(t), \Delta_{\exp }$ and $\Delta_{\text {sim }}$, respectively (see Table III). The Vandenboomgaerde $e t$ al. model prediction using the experimental parameters is in best agreement with the simulation amplitude, while the Zhang-Sohn Padé model prediction using the simulation parameters is in best agreement with the simulation amplitude. The Mikaelian bubble amplitude model prediction using the experimental parameters is in best agreement with the simulation bubble amplitude, while the Sadot et al. model prediction using the simulation parameters is in best agreement with the simulation bubble amplitude. The Sadot et al. spike amplitude model prediction using the experimental parameters is in best agreement with the simulation spike amplitude, while the Matsuoka et al. Padé model prediction using the simulation parameters is in best agreement with the simulation spike amplitude. Table III also shows the average fractional deviation between the experimental amplitude data $a_{\text {exp }}(t)$ and the model predictions $a_{\text {mod }}(t)$ when the model predictions are computed using the experimental and simulation parameters, $\Delta_{\exp }^{e}$ and $\Delta_{\text {sim }}^{e}$, respectively: the Sadot $e t$ al. Padé model prediction using the experimental parameters is in best agreement with the experimental amplitude, while the Zhang-Sohn Padé model prediction using the simulation parameters is in best agreement with the simulation amplitude. 

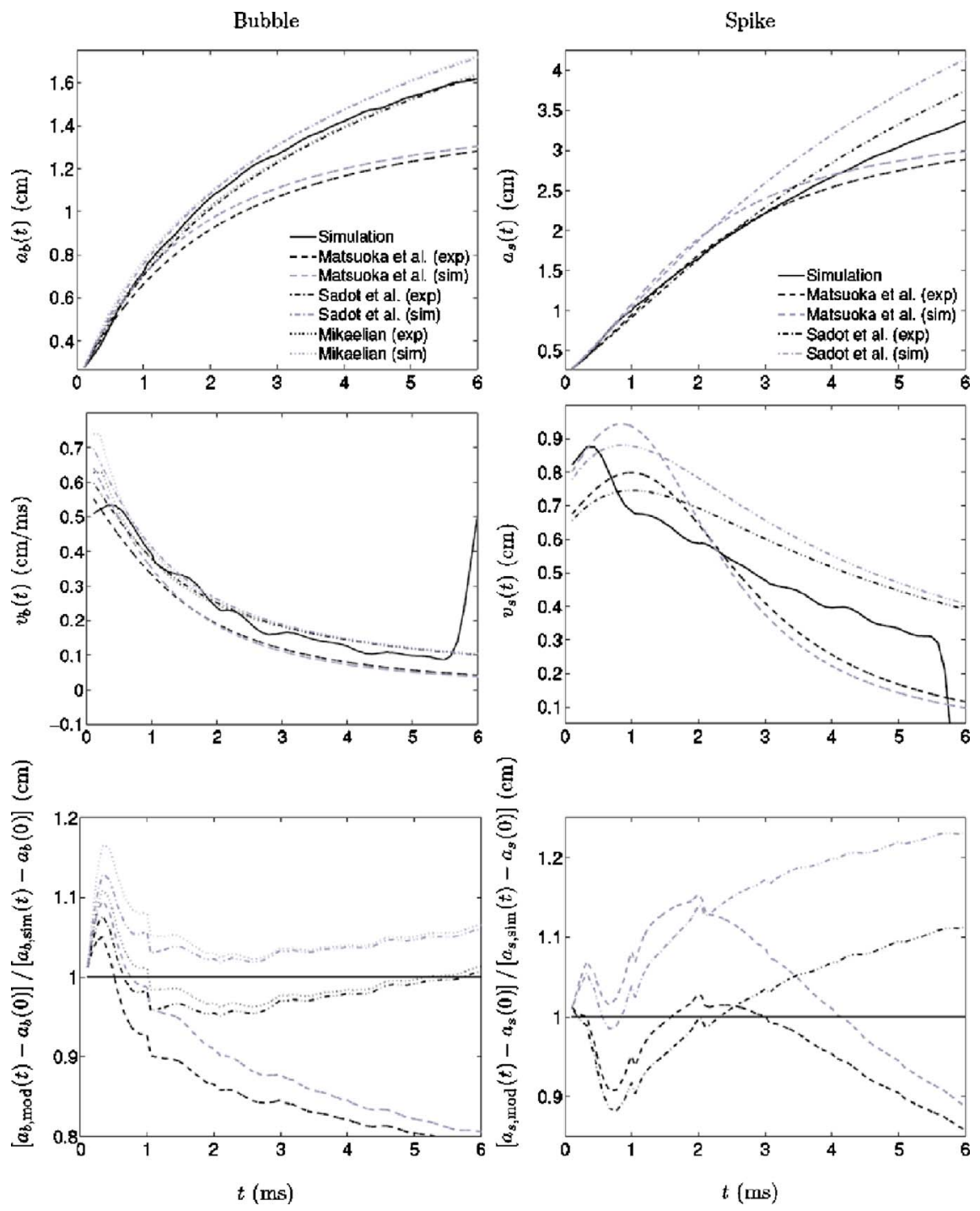

FIG. 14. The predictions of the bubble amplitude, bubble velocity, and ratio of the model predictions (with the initial perturbation amplitude subtracted out) to the ninth-order WENO simulation data, using the experimental and simulation parameters $v_{0}, A^{+}, a_{0}^{+}$, and $\Delta u$ (as given in Table II) (left column). The predictions of the spike amplitude, spike velocity, and ratio of the model predictions to the simulation results, using the experimental and simulation parameters, are also shown (right column).

\section{DISCUSSION AND CONCLUSIONS}

The high-resolution fifth- and ninth-order weighted essentially nonoscillatory (WENO) shock-capturing method was applied to the classical two-dimensional, reshocked single-mode Richtmyer-Meshkov instability with a uniform grid resolution corresponding to 256 points per initial perturbation wavelength. The initial conditions and computational domain approximated the Mach 1.21 air(acetone) $/ \mathrm{SF}_{6}$ shock tube experiment of Collins and Jacobs. ${ }^{15}$ Only the test section of the shock tube was simulated, so that the driver-based expansion and reflected rarefaction waves present in the experiment were not captured in the simulations. This is a convenient idealization, as the amplitude growth models considered do not account for these waves. A single value of the adiabatic exponent was used. Molecular dissipation and diffusion terms in the governing equations were neglected.

The simulation density and the experimental PLIF images from the experiment were in very good qualitative agreement up to the time at which the driver-based expansion affects the experimental amplitude growth (and before reshock when three-dimensional effects are unimportant). The comparison of densities from the fifth- and ninth-order simulations demonstrates that higher-order reconstruction better captures secondary instabilities, the roll-ups are tighter and sharper, and more fine-scale structures are present as a result of decreased numerical dissipation and diffusion. Following reshock, the experiment and simulations show distinctively different flow structures due to the absence of the driver-based expansion, the difference in the interface velocities, and the increased importance of three-dimensional effects not captured in the simulations. As a result, the simulation densities are qualitatively similar to the corrected experimental density PLIF images, but lag in time by $\approx 1 \mathrm{~ms}$.

Very good quantitative agreement was found between the fifth- and ninth-order amplitude and the experimental data points up to the time at which the driver-based expansion in the experiment (but not present in either the simulations or models) begins to expand the layer prior to reshock. A comparison of a large number of widely used models applied to the present simulations with the diffuse interface correction (35) was performed. The ninth-order simulation data were compared to the predictions of (i) the linear impulsive models of Richtmyer, ${ }^{1}$ Meyer and Blewett, ${ }^{44}$ 
Fraley, ${ }^{45}$ and Vandenboomgaerde et al. ${ }^{47}$ (ii) the weakly nonlinear perturbation models of Zhang and Sohn, ${ }^{48}$ Vandenboomgaerde et al. ${ }^{49}$ and Matsuoka et al. ${ }^{50}$ and (iii) the nonlinear empirical model of Sadot et $a .^{51}$ The bubble and spike velocities were also compared to the predictions of the potential models of Goncharov ${ }^{53}$ and Sohn. ${ }^{54,55}$ In addition, the bubble amplitude was compared to the prediction of the Mikaelian ${ }^{58}$ model. A local and a global metric was used to quantify the differences between the growth model predictions and between the simulation and experimental data-the ratio of the model predictions to the ninth-order amplitude as a function of time (local) and the average fractional deviation (global). As expected, very good agreement with the linear models was found at early times. At later times, the simulation data were shown to be in very good agreement with the predictions of the Zhang-Sohn Padé model before reshock. Thus, this component of the present work provides a validation of the WENO method against pre-reshock single-mode Richtmyer-Meshkov instability experimental data.

Motivated by the agreement between the simulation amplitudes and the experimental data, but the different conclusions regarding which amplitude growth model best agrees with the simulation and experimental data, the sensitivity of the model predictions to changes in the model parameters $v_{0}$, $A^{+}, a_{0}^{+}$, and $\Delta u$ was also investigated. First, the parameters from the Collins-Jacobs experiment and from the present simulation were compared. Then it was shown that the predictions of the nonlinear growth models are quite sensitive to variations in these parameters. In particular, when experimental parameters are used, the prediction of the Sadot et al. model is in best agreement with the experimental and simulation amplitude; by contrast, when the simulation parameters are used, the prediction of the Zhang-Sohn Padé model is in best agreement with the experimental and simulation amplitude. These results indicate that caution should be used when applying nonlinear amplitude growth models to experiments and to numerical simulations and interpreting their predictions. Moreover, it may not be possible to distinguish between the predictions of different models when the parameters are varied within the experimentally reported error bars.

While the present simulations are idealized in that they consider only the shock tube test section, two-dimensional flow, and a single gamma, the agreement with experimental data prior to reshock provides encouragement for the continued quantitative investigation of shock-induced complex hydrodynamic flows and mixing using high-order WENO methods. Other researchers have also advocated the WENO method for simulating compressible flows. ${ }^{64-68}$ The hybridization of WENO reconstructions with high-order centraldifference or spectral schemes ${ }^{69}$ to improve computational efficiency and reduce numerical dissipation in regions away from discontinuities is currently under development and will be used in future investigations of the physics of RichtmyerMeshkov mixing.

\section{ACKNOWLEDGMENTS}

The authors thank Dr. Jeffrey W. Jacobs for clarifying aspects of the experiment considered in this work, Dr. Vitaliy
V. Krivets for providing details not originally presented in the description of the experiments, and Dr. Karnig O. Mikaelian for useful comments on the perturbation amplitude models. M.L. acknowledges support from the Air Force Office of Scientific Research through the National Defense Science and Engineering Graduate Fellowship and the Caltech Accelerated Strategic Computing (ASC) Center. W.S.D. acknowledges support of this work by the DOE under Contract No. DE-FG02-98ER25346 and the AFOSR under Contract No. FA9550-05-1-0123. This work was also performed under the auspices of the U.S. Department of Energy by the University of California, Lawrence Livermore National Laboratory under Contract No. W-7405-Eng-48.

${ }^{1}$ R. D. Richtmyer, "Taylor instability in shock acceleration of compressible fluids," Commun. Pure Appl. Math. 8, 297 (1960).

${ }^{2}$ E. E. Meshkov, "Instability of the interface of two gases accelerated by a shock wave," Fluid Dyn. 4, 101 (1969).

${ }^{3}$ N. J. Zabusky, "Vortex paradigm for accelerated inhomogeneous flows: Visiometrics for the Rayleigh-Taylor and Richtmyer-Meshkov environments," Annu. Rev. Fluid Mech. 31, 495 (1999).

${ }^{4}$ M. Brouillette, "The Richtmyer-Meshkov instability," Annu. Rev. Fluid Mech. 34, 445 (2002).

${ }^{5}$ J. D. Lindl, "Development of the indirect-drive approach to inertial confinement fusion and the target physics basis for ignition and gain," Phys. Plasmas 2, 3933 (1995).

${ }^{6} \mathrm{~S}$. Nakai and H. Takabe, "Principles of inertial confinement fusionphysics of implosion and the concept of inertial fusion energy," Rep. Prog. Phys. 59, 1071 (1996).

${ }^{7}$ J. D. Lindl, Inertial Confinement Fusion: The Quest for Ignition and Energy Gain Using Indirect Drive (AIP, New York, 1998).

${ }^{8}$ S. Atzeni and J. Meyer-ter-Vehn, The Physics of Inertial Confinement Fusion: Beam Plasma Interaction, Hydrodynamics, Hot Dense Matter, Vol. 125 of International Series of Monographs on Physics (Oxford University Press, New York, 2004).

${ }^{9}$ G. J. Hendricks, "Two mechanisms of vorticity generation in combusting flow fields," Ph.D. thesis, California Institute of Technology (1986).

${ }^{10}$ S. W. Falk and W. D. Arnett, "A theoretical model for type II supernovae," Astrophys. J., Lett. 180, L65 (1973).

${ }^{11}$ W. D. Arnett, "Supernova theory and supernova 1987A," Astrophys. J. 319, 136 (1987).

${ }^{12}$ W. D. Arnett, B. A. Fryxell, and E. Müller, "Instabilities and nonradial motion in SN-1987A," Astrophys. J. Lett. 341, L63 (1989).

${ }^{13}$ J. Kane, R. P. Drake, and B. A. Remington, "An evaluation of the Richtmyer-Meshkov instability in supernova remnant formation," Astrophys. J. 511, 335 (1999).

${ }^{14}$ D. Arnett, "The role of mixing in astrophysics," Astrophys. J., Suppl. Ser. 127, 213 (2000).

${ }^{15} \mathrm{~B}$. D. Collins and J. W. Jacobs, "PLIF flow visualization and measurements of the Richtmyer-Meshkov instability of an air/SF$F_{6}$ interface," J. Fluid Mech. 464, 113 (2002).

${ }^{16}$ G. Peng, N. J. Zabusky, and S. Zhang, "Vortex-accelerated secondary baroclinic vorticity deposition and late-intermediate time dynamics of a two-dimensional Richtmyer-Meshkov interface," Phys. Fluids 15, 3730 (2003).

${ }^{17}$ P. E. Dimotakis, "The mixing transition in turbulent flows," J. Fluid Mech. 409, 69 (2000).

${ }^{18}$ K. O. Mikaelian, "Numerical simulations of Richtmyer-Meshkov instabilities in finite-thickness fluid layers," Phys. Fluids 8, 1269 (1996).

${ }^{19}$ O. Sadot, L. Erez, G. Oron, G. Ben-Dor, U. Alon, L. A. Levin, and D. Shvarts, "Studies on the nonlinear evolution of the Richtmyer-Meshkov instability," Astrophys. J., Suppl. Ser. 127, 469 (2000).

${ }^{20}$ J. W. Jacobs, M. A. Jones, and C. E. Niederhaus, "Experimental studies of Richtmyer-Meshkov instability," in Proceedings of the Fifth International Workshop on Compressible Turbulent Mixing, edited by R. Young, J. Glimm, and B. Boston (World Scientific, New York, 1996), pp. 195-202.

${ }^{21}$ C. E. Niederhaus and J. W. Jacobs, "Experimental study of the RichtmyerMeshkov instability of incompressible fluids," J. Fluid Mech. 485, 243 (2003). 
${ }^{22}$ A. D. Kotelnikov and N. J. Zabusky, "Vortex dynamics of a twiceaccelerated interface in an incompressible ideal fluid," Astrophys. J., Suppl. Ser. 127, 389 (2000)

${ }^{23}$ A. D. Kotelnikov, J. Ray, and N. J. Zabusky, "Vortex morphologies on reaccelerated interfaces: Visualization, quantification and modeling of one- and two-mode compressible and incompressible environments," Phys. Fluids 12, 3245 (2000)

${ }^{24}$ J. W. Jacobs, D. G. Jenkins, D. L. Klein, and R. F. Benjamin, "Nonlinear growth of the shock-accelerated instability of a thin fluid layer," J. Fluid Mech. 295, 23 (1995)

${ }^{25}$ P. M. Rightley, P. Vorobieff, and R. F. Benjamin, "Evolution of a shockaccelerated thin fluid layer," Phys. Fluids 9, 1770 (1997).

${ }^{26}$ K. Kremeyer, S. Nazarenko, and A. C. Newell, "Shock bowing and vorticity dynamics during propagation into different transverse density profiles," Physica D 163, 150 (2002).

${ }^{27}$ S. Zhang, N. J. Zabusky, G. Peng, and S. Gupta, "Shock gaseous cylinder interactions: Dynamically validated initial conditions provide excellent agreement between experiments and numerical simulations to lateintermediate time," Phys. Fluids 16, 1203 (2004).

${ }^{28}$ C. B. Laney, Computational Gas Dynamics (Cambridge University Press, New York, 1998)

${ }^{29}$ R. J. Leveque, Finite Volume Methods for Hyperbolic Problems, Vol. 31 of Cambridge Texts in Applied Mathematics (Cambridge University Press, New York, 2002).

${ }^{30}$ A. J. Chorin and J. E. Marsden, A Mathematical Introduction to Fluid Mechanics, Vol. 4 of Texts in Applied Mathematics, 3rd ed. (SpringerVerlag, New York, 1993).

${ }^{31}$ J. P. Boris, F. F. Grinstein, E. S. Oran, and R. L. Kolbe, "New insights into large eddy simulation," Fluid Dyn. Res. 10, 199 (1992).

${ }^{32}$ D. Drikakis, "Advances in turbulent flow computations using highresolution methods," Prog. Aerosp. Sci. 39, 405 (2003).

${ }^{33}$ M. Latini, O. Schilling, and W. S. Don, "Effects of WENO flux reconstruction order and spatial resolution on reshocked two-dimensional Richtmyer-Meshkov instability,” J. Comput. Phys. 221, 805 (2007).

${ }^{34}$ D. Balsara and C.-W. Shu, "Monotonicity preserving weighted essentially non-oscillatory schemes with increasingly high order of accuracy," J. Comput. Phys. 160, 405 (2000).

${ }^{35}$ C.-W. Shu, "High order ENO and WENO schemes for computational fluid dynamics," in High-Order Methods for Computational Physics, edited by T. J. Barth and H. Deconinck (Springer-Verlag, New York, 1999), pp. 439-582.

${ }^{36}$ S. Karni, "Multicomponent flow calculations by a consistent primitive algorithm," J. Comput. Phys. 112, 31 (1994).

${ }^{37}$ S. Karni, "Hybrid multifluid algorithms," SIAM J. Sci. Comput. 17, 1019 (1996).

${ }^{38} \mathrm{R}$. Abgrall, "How to prevent pressure oscillations in multicomponent flow calculations: A quasi-conservative approach," J. Comput. Phys. 125, 150 (1996).

${ }^{39}$ R. Abgrall and S. Karni, "Computations of compressible multifluids," J. Comput. Phys. 169, 594 (2001).

${ }^{40}$ C.-W. Shu and S. Osher, "Efficient implementation of essentially nonoscillatory shock-capturing schemes," J. Comput. Phys. 77, 439 (1988).

${ }^{41}$ M. A. Jones and J. W. Jacobs, "A membraneless experiment for the study of Richtmyer-Meshkov instability of a shock-accelerated gas interface," Phys. Fluids 9, 3078 (1997).

${ }^{42}$ J. W. Jacobs and V. V. Krivets, "Experiments on the late-time development of single-mode Richtmyer-Meshkov instability," Phys. Fluids 17, 034104 (2005).

${ }^{43}$ G. I. Taylor, "The instability of liquid surfaces when accelerated in a direction perpendicular to their planes," Proc. R. Soc. London, Ser. A 201, $192(1950)$

${ }^{44}$ K. A. Meyer and P. J. Blewett, "Numerical investigation of the stability of a shock accelerated interface between two fluids," Phys. Fluids 15, 753 (1972).

${ }^{45}$ G. Fraley, "Rayleigh-Taylor stability for a normal shockwave-density discontinuity interaction," Phys. Fluids 29, 376 (1986).

${ }^{46}$ K. O. Mikaelian, "Freeze-out and the effect of compressibility in the Richtmyer-Meshkov instability," Phys. Fluids 6, 356 (1994).

${ }^{47}$ M. Vandenboomgaerde, C. Mügler, and S. Gauthier, "Impulsive model for the Richtmyer-Meshkov instability," Phys. Rev. E 58, 1874 (1998).

${ }^{48} \mathrm{Q}$. Zhang and S. Sohn, "Non-linear theory of unstable fluid mixing driven by shock wave," Phys. Fluids 9, 1106 (1997).

${ }^{49}$ M. Vandenboomgaerde, S. Gauthier, and C. Mügler, "Nonlinear regime of a multimode Richtmyer-Meshkov instability: A simplified perturbation theory," Phys. Fluids 14, 1111 (2002).

${ }^{50}$ C. Matsuoka, K. Nishihara, and Y. Fukuda, "Nonlinear evolution of an interface in the Richtmyer-Meshkov instability," Phys. Rev. E 67, 036301 (2003); erratum, ibid. 68, 029902 (2003).

${ }^{51}$ O. Sadot, L. Erez, U. Alon, D. Oron, and L. A. Levin, "Study of nonlinear evolution of single-mode and two bubble interaction under RichtmyerMeshkov instability," Phys. Rev. Lett. 80, 1654 (1998).

${ }^{52}$ D. Layzer, "On the instability of superposed fluids in a gravitational field," Astrophys. J. 122, 1 (1955).

${ }^{53} \mathrm{~V}$. N. Goncharov, "Analytical model of nonlinear, single-mode, classical Rayleigh-Taylor instability at arbitrary Atwood numbers," Phys. Rev. Lett. 88, 134502 (2002).

${ }^{54}$ S.-I. Sohn, "Simple potential-flow model of Rayleigh-Taylor and Richtmyer-Meshkov instabilities for all density ratios,” Phys. Rev. E 67, 026301 (2003).

${ }^{55}$ S.-I. Sohn, "Density dependence of a Zufiria-type model for RayleighTaylor bubble fronts," Phys. Rev. E 70, 045301 (2004).

${ }^{56}$ J. A. Zufiria, "Bubble competition in Rayleigh-Taylor instability," Phys. Fluids 31, 440 (1988)

${ }^{57}$ S.-I. Sohn and Q. Zhang, "Late time behavior of bubbles at unstable interfaces in two dimensions," Phys. Fluids 13, 3493 (2001).

${ }^{58}$ K. O. Mikaelian, "Explicit expressions for the single-mode RayleighTaylor and Richtmyer-Meshkov instabilities at arbitrary Atwood numbers," Phys. Rev. E 67, 026319 (2003).

${ }^{59}$ H. M. Antia, Numerical Methods for Scientists and Engineers (Birkäuser, Boston, 2002).

${ }^{60}$ A. W. Cook and P. E. Dimotakis, "Transition stages of Rayleigh-Taylor instability between miscible fluids," J. Fluid Mech. 443, 69 (2001); corrigendum, ibid. 457, 410 (2002).

${ }^{61}$ L. F. Henderson, "On the refraction of shock waves," J. Fluid Mech. 198, 365 (1989)

${ }^{62}$ K. O. Mikaelian, "Density gradient stabilization of the RichtmyerMeshkov instability," Phys. Fluids A 3, 2638 (1991).

${ }^{63}$ R. E. Duff, F. H. Harlow, and C. W. Hirt, "Effects of diffusion on interface instability between gases," Phys. Fluids 5, 417 (1962).

${ }^{64}$ K. Sinha and G. V. Candler, "Turbulent dissipation-rate equation for compressible flows," AIAA J. 41, 1017 (2003).

${ }^{65}$ S. Pirozzoli, F. Grasso, and T. B. Gatski, "Direct numerical simulation and analysis of a spatially evolving supersonic turbulent boundary layer at $M=2.25$," Phys. Fluids 16, 530 (2004).

${ }^{66} \mathrm{~J}$. A. Ekaterinaris, "Performance of high-order-accurate, low-diffusion numerical schemes for compressible flow," AIAA J. 42, 493 (2004).

${ }^{67} \mathrm{~J}$. A. Ekaterinaris, "High-order accurate, low numerical diffusion methods for aerodynamics," Prog. Aerosp. Sci. 41, 192 (2005).

${ }^{68}$ T. S. Cheng and K. S. Lee, "Numerical simulations of underexpanded supersonic jet and free shear layer using WENO schemes," Int. J. Heat Fluid Flow 26, 755 (2005).

${ }^{69}$ B. Costa, W. S. Don, D. Gottlieb, and R. Sendersky, "Two-dimensional multi-domain hybrid spectral-WENO methods for conservation laws," Commun. Comput. Phys. 1, 548 (2006). 